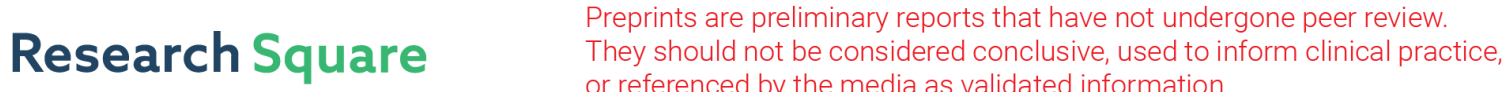 or referenced by the media as validated information. \\ Cyclic Experiments and Global Buckling Design of Steel-Angles-Assembled Buckling-Restrained Braces
}

\section{Jing-Zhong Tong ( $\nabla$ tongjz@zju.edu.cn )}

Zhejiang University https://orcid.org/0000-0003-3190-518X

\section{En-Yuan Zhang}

Zhejiang University

\section{Yan-Lin Guo}

Tsinghua University

Chao-Qun Yu

Zhejiang University

\section{Research Article}

Keywords: Steel-angels-assembled buckling-restrained braces, Hysteretic behavior, Cyclic experiments, Finite element analysis, Restraining ratio, Global buckling design

Posted Date: December 30th, 2021

DOI: https://doi.org/10.21203/rs.3.rs-1204081/v1

License: (9) This work is licensed under a Creative Commons Attribution 4.0 International License. Read Full License

Version of Record: A version of this preprint was published at Bulletin of Earthquake Engineering on April 4th, 2022. See the published version at https://doi.org/10.1007/s10518-022-01389-w. 


\title{
Cyclic experiments and global buckling design of steel-angles-assembled buckling-restrained braces
}

\author{
Jing-Zhong Tong a,b, ${ }^{*}$, En-Yuan Zhang a , Yan-Lin Guo ${ }^{\text {b }}$, Chao-Qun Yu ${ }^{\text {a }}$ \\ ${ }^{a}$ Institute of Advanced Engineering Structures and Materials, Zhejiang University, Hangzhou 310058, China \\ ${ }^{b}$ Department of Civil Engineering, Tsinghua University, Beijing 100084, China
}

Abstract: Compared with traditional BRB, the steel-angles-assembled buckling-restrained brace (SAA-BRB) is an innovative BRB with light-weight, accurate control of the geometrical dimensions, easy installation and convenient disassembly. The SAA-BRB is composed of an external restraining system and a cruciform-sectional inner core. The external restraining system is assembled by four steel angles with the connection of high-strength bolts, and the spacers are installed between the inner core and the restraining system. In this study, the hysteretic behavior of SAA-BRB was investigated by experiments and finite element (FE) simulations. Firstly, three SAA-BRB specimens with different restraining ratios were tested under cyclic loads to investigate the hysteretic performance. It was found that all specimens exhibited stable responses and satisfactory energy-dissipating capabilities during the whole loading process. Then, a refined FE model was established, and its validity in predicting the hysteretic responses of SAA-BRB was verified by the experiments. Moreover, based on the yielding criteria of the outmost fiber for the restraining member section, a design formula for the restraining ratio requirements to avoid global buckling of the SAA-BRB was deduced. Finally, extensive parametric analysis was conducted to verify the accuracy of the design formula by changing the geometric dimensions (the restraining ratio) of models. It was found that the proposed formula for the restraining ratio requirement could lead to a conservative prediction with reasonable accuracy, thus providing valuable references for global buckling design of SAA-BRBs in engineering practice.

Keywords: Steel-angels-assembled buckling-restrained braces; Hysteretic behavior; Cyclic experiments; Finite element analysis; Restraining ratio; Global buckling design

\footnotetext{
* Corresponding author

E-mail address: tongjz@zju.edu.cn
} 


\section{Nomenclature}

Symbol Description

$A_{1} \quad$ Cross-sectional area of a single steel angle

$A_{0} \quad$ Cross-sectional area of the external restraining system

$A_{\mathrm{c}} \quad$ Cross-sectional area of the steel core

$b \quad$ Width of yield segment of the inner core

$b_{\mathrm{a}} \quad$ Length of the steel angle flange

$b_{1} \quad$ Width of strengthened segment of the inner core

$f_{\mathrm{yr}} \quad$ Yield stress of the external restraining system

$f_{\mathrm{yc}} \quad$ Yield stress of the inner steel core

$F_{\mathrm{y}} \quad$ Yield load of the specimen

$F_{\max } \quad$ Maximum load of the specimen

$g$

Gap size between the steel core and restraining system

$h_{\mathrm{ar}} \quad$ Distance between the edge of restraining system and the bolts circle center

$H \quad$ Height of frame connected with the BRB

$I_{0} \quad$ Moment of inertia regarding the external restraining system as a whole

$I_{1} \quad$ Moment of inertia of a single steel angle

$K_{\mathrm{i}} \quad$ Axial secant stiffness

$l \quad$ Length of SAA-BRB

$l_{0} \quad$ Length of the restraining system

$l_{\mathrm{y}} \quad$ Length of yield segment of the inner core

$l_{\mathrm{u}} \quad$ Length of unrestrained segment of the inner core

$l_{\mathrm{t}} \quad$ Length of transition segment of the inner core

$l_{\mathrm{s}} \quad$ Length of strengthened segment of the inner core

$\Delta l_{\mathrm{y}} \quad$ Axial deformation of the BRB

$\Delta_{\mathrm{y}} \quad$ Yield displacement of the specimen

$\Delta_{\max } \quad$ Maximum displacement of the specimen

$+\Delta_{\mathrm{i}} \quad$ Displacement corresponding to tensile peak point at $\mathrm{i}$-th hysteretic cycle 
$-\Delta_{\mathrm{i}} \quad$ Displacement corresponding to compressive peak point at $\mathrm{i}$-th hysteretic cycle

$l_{1} \quad$ Bolts spacing along the longitudinal direction

$L \quad$ Length of frame connected with BRB

$M_{\max } \quad$ Maximum bending moment at mid-span of the restraining system

$P_{\text {cr }} \quad$ Euler buckling load of BRB

$P_{\mathrm{cr}, 0} \quad$ Euler buckling load of the external restraining system of ordinary BRB

$P_{\mathrm{cr}, \mathrm{c}} \quad$ Euler buckling load of the inner core

$P_{\mathrm{cr}, \mathrm{r}} \quad$ Euler buckling load of the external restraining system of SAA-BRB

$P_{\mathrm{y}} \quad$ Yield load of the inner core

$P_{\text {cmax }} \quad$ Load corresponding to compressive peak point of the hysteretic curve

$P_{\operatorname{tmax}} \quad$ Load corresponding to tensile peak point of the hysteretic curve

$+P_{\mathrm{i}} \quad$ Load corresponding to tensile peak point at i-th hysteretic cycle

$-P_{\mathrm{i}} \quad$ Load corresponding to compressive peak point at i-th hysteretic cycle

$S_{(\mathrm{ABC}+\mathrm{CDA})} \quad$ Area enclosed by a single hysteretic curve and the abscissa

$S_{\text {(OBE+ODF) }} \quad$ Triangle area enclosed by a dotted line and the abscissa

$t$

Thickness of the inner core

$t_{\mathrm{a}} \quad$ Thickness of steel angle

Amplitude of the initial geometrical imperfection of the SAA-BRB

$v_{\max }$

Lateral maximum deformation at mid-span of the restraining system

$W$

Plastic section modulus of the external restraining system of SAA-BRB

$\omega$

Reduction factor of global buckling load of external restraining system

Restraining ratio

[ל]

Restraining ratio requirements

$\lambda_{0}$

Slenderness ratio regarding the external restraining system as a whole

$\lambda_{1}$

Slenderness ratio of a segment of single chord member

$\sigma_{\max }$

Maximum normal stress of the external restraining system

$\lambda_{\mathrm{c}}$

Slenderness ratio of the inner core

$\alpha$

Angle between the BRB and its connected frame beam 
$\left[\varepsilon_{\mathrm{c}}\right] \quad$ Maximum axial strain requirement of the BRB

$\varphi \quad$ Ratio of the inner core yield segment length to BRB length

$\beta \quad$ Compression strength adjustment factor

$\xi_{\mathrm{eq}} \quad$ Equivalent viscous damping ratio

28 


\section{Introduction}

As a high-performance member with excellent lateral resistance and energy dissipation capacity, buckling-restrained brace (BRB) has been widely used in engineering practice in many countries such as Japan, the US, and China over the years (Kiggins and Uang 2006; Tremblay et al. 2006; Zhao et al. 2017). Generally, the BRB is composed of an inner steel core encased by an external restraining system. The inner core is used to bear the axial load, and the external member is used to limit the lateral displacement of the inner core to prevent it from global buckling (Tong et al. 2020). In the design of BRB, the inner core should reach its fullsectional yield load without undergoing global buckling/local failure mode, ensuring the excellent load-carrying performance and energy-dissipating capacity of BRBs (Black et al. 2004; Eryasar and Topkaya 2010; Kiggins and Uang 2006; Tong and Guo 2018).

Under regular use or minor earthquakes, BRB can provide sufficient lateral stiffness and horizontal bearing capacity for the frame structure as common braces, and the inner core is in an elastic working state. Under the action of severe earthquakes, BRB can fully develop its plasticity and energy dissipation capacity, reducing damage to the main frame structure (Ariyaratana and Fahnestock 2011; Takeuchi et al. 2010; Zhao et al. 2013). Therefore, the application of BRB not only avoids the global bucking of ordinary braces under severe earthquakes, but achieves excellent energy-dissipating performance to protect the frame structure (Shi et al. 2018).

The concept of BRB was first proposed by Yoshino and Karino (1971). In that study, a member with steel plates embedded in reinforced concrete shear walls was proposed. Its working principle is to restrain the buckling of the embedded steel plate through the external concrete wall panel. However, this is not conductive to the material utilization because only the diagonal strip-shaped area of the concrete wall can directly limit the lateral deformation of the embedded steel plate. In this consideration, BRB has gradually evolved into a type in which

54 a slender external restraining system constrains the inner core to further improve the utilization of material. In early development stage of this type of BRBs, a typical restraining member is 
al. 1992). Since the restraining system is used to limit the lateral deformation of the inner core, a thin layer of unbonded material should be installed at the interface between the inner core and its surrounding concrete to avoid axial force transmission (Fujimoto et al. 1990; Ju et al. 2009; Palazzo et al. 2009). However, complex construction procedures caused by wet concrete work, heavy self-weight and inconvenient disassembly result in difficulties when repairing the BRB after an earthquake (Ju et al. 2009; Shi et al. 2018). To solve these problems, researchers proposed BRBs with all-steel composition and conducted experimental and numerical studies (Guo et al. 2016; Guo et al. 2017a, b; Hosseinzadeh and Mohebi 2016; Jia et al. 2017; Judd et al. 2016; Mirtaheri et al. 2017). The all-steel BRB is mainly characterized by a steel core with a circular section or $\mathrm{H}$-section and a restraining member of a circular or square steel tube. Due to its all-steel composition, there is no need for unbonded material at the interface between the inner core and the restraining member as an air gap could be precisely controlled during its fabrication (Guo et al. 2017a; Jia et al. 2017; Mirtaheri et al. 2017). Besides, the self-weight of all-steel BRB is significantly reduced compared with the traditional BRB composed of an inner steel core with the encasement of concrete-filled steel tube. Furthermore, to satisfy the requirements of high-rise buildings or long-span structures with popularity in recent years, assembled BRBs with bolt-connected restraining systems were proposed and investigated by numerous researchers (Chou and Chen 2010; Dehghani and Tremblay 2018; Ding 2014; Guo et al. 2018; Jiang et al. 2017; Metelli et al. 2016; Tong and Guo 2018; Chou et al. 2019). Chou and Chen (2010) developed a sandwiched assembled BRB with an inner core encased by two identical restraining members connected with high-strength bolts, and four BRB specimens were tested to investigate the load-carrying performance. Dehghani and Tremblay (2018) proposed an all-steel assembled BRB composed of a flat plate core and a rectangular restraining member with a hollow steel section. The stable seismic performance and high post-yield stiffness of this BRB were examined by experiments. Jiang et al. (2017) developed a pinned double-rectangular tube assembled BRB, which exhibited satisfactory energy-dissipating performance under repeated axial-tension loading. Guo et al. (2018) proposed an assembled 
model that could well predict the hysteretic responses was established and a design procedure was proposed subsequently. For assembled BRBs, the external restraining system generally includes multiple components connected by high-strength bolts. The advantages of assembled BRB include: (1) the fabrication of BRB with the bolt connection is convenient and quick; (2) the BRB can be easily disassembled, reducing the difficulty in transportation and erection; (3) the damaged core can be easily replaced in the post-earthquake structure due to the feature of easy disassembling. It should be noted that the discrete arrangement of the connecting bolts will weaken the rigidity of the restraining member in the design of assembled BRBs.

In this paper, a new type of assembled BRB called steel-angles-assembled BRB (SAABRB) was studied. The SAA-BRB consists of a cruciform-sectional steel core and a restraining system composed of four steel angles. The cyclic loading experiment was carried out on three specimens to investigate the load-carrying performance and hysteretic response of SAA-BRB. Besides, a finite element (FE) model of SAA-BRB was established, and the comparison against experimental results verified the feasibility of the model. Moreover, the theoretical design formula with restraining ratio requirements to prevent SAA-BRB from global buckling was derived. Finally, extensive FE simulations were performed to validate the proposed design formula.

\section{Cyclic experiments}

\subsection{Experimental specimens}

Three SAA-BRB specimens were designed to experimentally investigate their hysteretic responses. Fig. 1 shows the construction details of SAA-BRB specimens, which consists of an inner steel core with a uniform cruciform-section and a restraining system as well as spacers.

The external restraining system is composed of four steel angles assembled by high-strength bolts (M16). The spacers with holes for the bolts to pass through are installed between the steel angles. The inner core can be divided into yield segment and unrestrained segment composed of a transition segment and a strengthened segment along its longitudinal direction. The unrestrained segment of the core was enhanced at both ends by expanding its cross-sectional size, and it was welded with a flange to prevent it from local buckling. Besides, the stiffened 
113 plates were set at the end of each steel angle along the longitudinal direction to enhance the

114 integrity of the external restraining member. According to Chinese design code GB50017 (GB

115 2017), the slenderness ratio of the core $\lambda_{\mathrm{c}}$ should be greater than $5.07 b / t$ to prevent the torsional

116 buckling failure of SAA-BRB. Additionally, the stoppers (i.e. displacement limitation fastener)

117 were designed and arranged on each side of the mid-span of the core by welding to inhibit the

118 restraining system from sliding longitudinally along the core.

119 Specimens S-1, S-2 and S-3 were similar in geometrical configuration, and the design

120 parameters of each specimen are tabulated in Table 1. For all specimens, SAA-BRB length, $l$,

121 and restraining system length, $l_{0}$, were $2400 \mathrm{~mm}$ and $2100 \mathrm{~mm}$, respectively. The steel angle

122 width $b_{\mathrm{a}}$ of Specimen S-1 was $80 \mathrm{~mm}$, and those of Specimens S-2 and S-3 were $90 \mathrm{~mm}$. Steel

123 angle thickness $t_{\mathrm{a}}$ was $6 \mathrm{~mm}$ for each specimen. The cruciform-sectional core member width,

$124 b$, was $100 \mathrm{~mm}$ for Specimens S-1 and S-2 while $110 \mathrm{~mm}$ for Specimen S-3. Inner core

125 thickness, $t$, was $22 \mathrm{~mm}$ for each specimen.

126 To avoid the axial load sustained by the inner core being transmitted to the restraining

127 system, hard rubbers with a thickness of $1.2 \mathrm{~mm}$ were installed between the steel core and four

128 steel angles. The spacer thickness of all specimens was $25 \mathrm{~mm}$, and the spacer widths in the

129 transverse direction of the cross-section and the longitudinal direction were $48 \mathrm{~mm}$ and $42 \mathrm{~mm}$,

130 respectively.

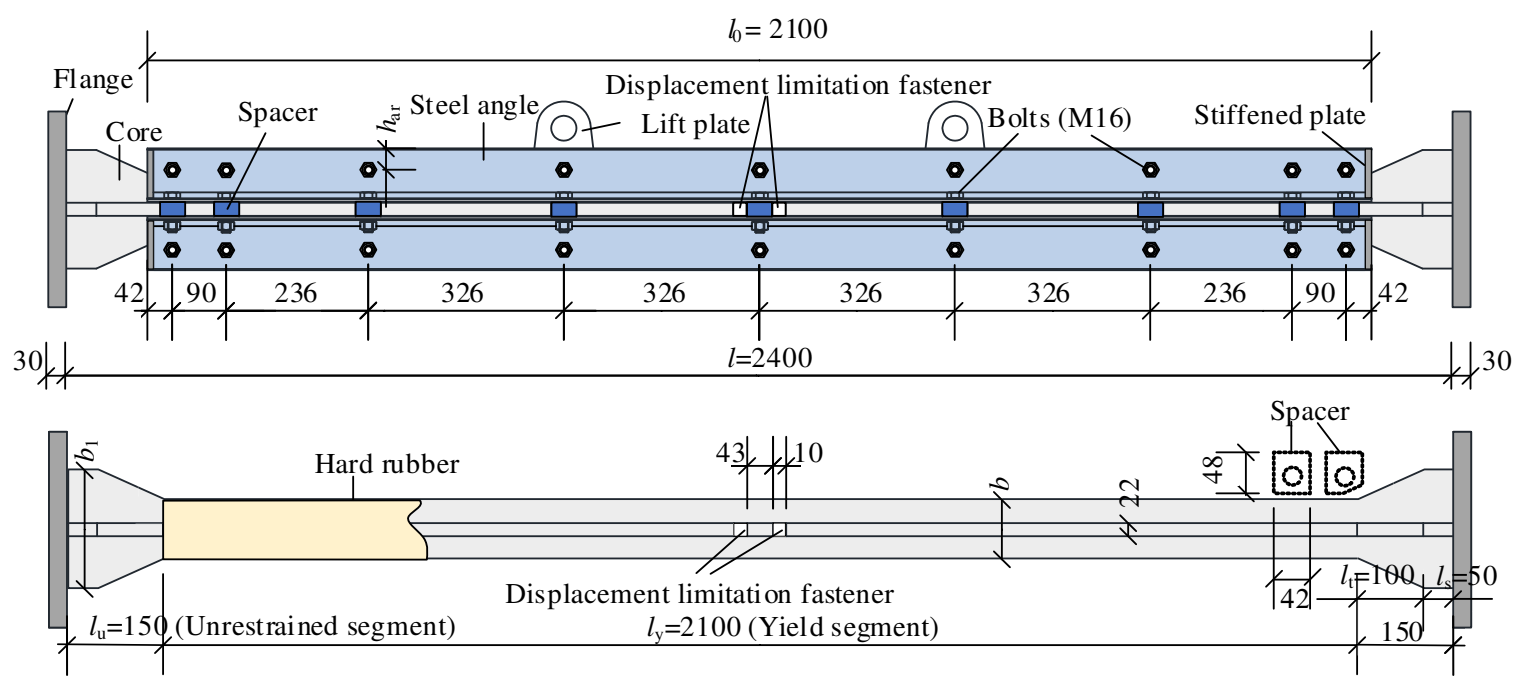

Fig. 1 Construction details of SAA-BRB specimens 
Table 1 Design parameters of SAA-BRB specimens

\begin{tabular}{cccccccccc}
\hline $\begin{array}{c}\text { Specimen } \\
\text { no. }\end{array}$ & $\begin{array}{c}b \\
(\mathrm{~mm})\end{array}$ & $\begin{array}{c}b_{1} \\
(\mathrm{~mm})\end{array}$ & $\begin{array}{c}b_{\mathrm{a}} \times t_{\mathrm{a}} \\
(\mathrm{mm})\end{array}$ & $\begin{array}{c}h_{\mathrm{ar}} \\
(\mathrm{mm})\end{array}$ & $\begin{array}{c}g \\
(\mathrm{~mm})\end{array}$ & $l_{0}$ & $\begin{array}{c}f_{\mathrm{yr}} \\
(\mathrm{MPa})\end{array}$ & $\begin{array}{c}f_{\mathrm{yc}} \\
(\mathrm{MPa})\end{array}$ & $\zeta$ \\
\hline S-1 & 100 & 200 & $80 \times 6$ & 25 & 1 & 2100 & 380 & 288 & 1.86 \\
$\mathrm{~S}-2$ & 100 & 200 & $90 \times 6$ & 35 & 1 & 2100 & 380 & 288 & 2.47 \\
$\mathrm{~S}-3$ & 110 & 220 & $90 \times 6$ & 35 & 1 & 2100 & 380 & 288 & 2.22 \\
\hline
\end{tabular}

2.2 Experimental setup and loading protocol

134 The MTS hydraulic servo testing machine was used for applying cyclic loads. Fig. 2 presents the experimental setup consisting of two reaction frames anchored to the strong floor and test specimens loaded via a $600 \mathrm{t}$ actuator with an in-line load cell. At both ends of each specimen, the flange with Q345 steel plate is connected to the cross-shaped connecting plate using high-strength bolts, and the cruciform-shaped connecting plate is also connected to the actuator and the reaction frame with the connection of bolts.

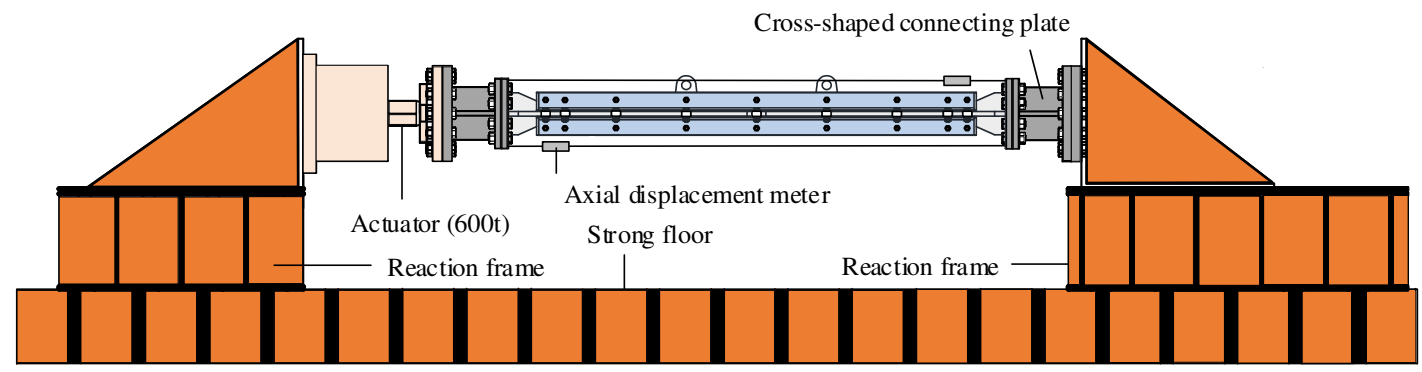

Fig. 2 Experimental setup

140 As shown in Fig. 3, $H$ and $L$ respectively denote the height and length of the frame connected with $\mathrm{BRB}, \alpha$ the angle between the $\mathrm{BRB}$ and the frame beam, thus the length of the

142 BRB $l$ can be expressed as $l=\sqrt{H^{2}+L^{2}}$. For BRB, the length of the yield segment of the core is

$143 l_{\mathrm{y}}$, thus the ratio of the core yield segment length to BRB length is denoted as $\varphi=l_{\mathrm{y}} / l$. According

144 to the Chinese code GB50010 (GB 2010) for seismic design of buildings, the maximum drift 145 angle of the frame structure is $1 / 50$. Hence, the maximum axial deformation of the inner core 146 can be expressed as $\Delta l_{y}=H \cos \alpha / 50$. In engineering practice, axial deformation mainly occurs in 147 the yield segment of the core due to the unrestrained segment of the core being enhanced by 148 increasing its sectional size. Therefore, the maximum axial strain requirement $\left[\varepsilon_{\mathrm{c}}\right]$ of the BRB 149 can be expressed as Eq. (1) by assuming that the axial deformation entirely occurs in the yield 
segment of the core.

$$
\left[\varepsilon_{\mathrm{c}}\right]=\frac{\Delta l_{\mathrm{y}}}{l_{\mathrm{y}}}=\frac{H \cos \alpha / 50}{\varphi H / \sin \alpha}=\frac{\sin \alpha \cos \alpha}{50 \varphi}
$$

151 Assuming that $\alpha=45^{\circ}$ and $\varphi=0.5$, it is calculated as $\left[\varepsilon_{\mathrm{c}}\right]=0.02$. Therefore, the axial strain of $152 \quad 2.0 \%$ was determined as the maximum strain in the loading protocol.
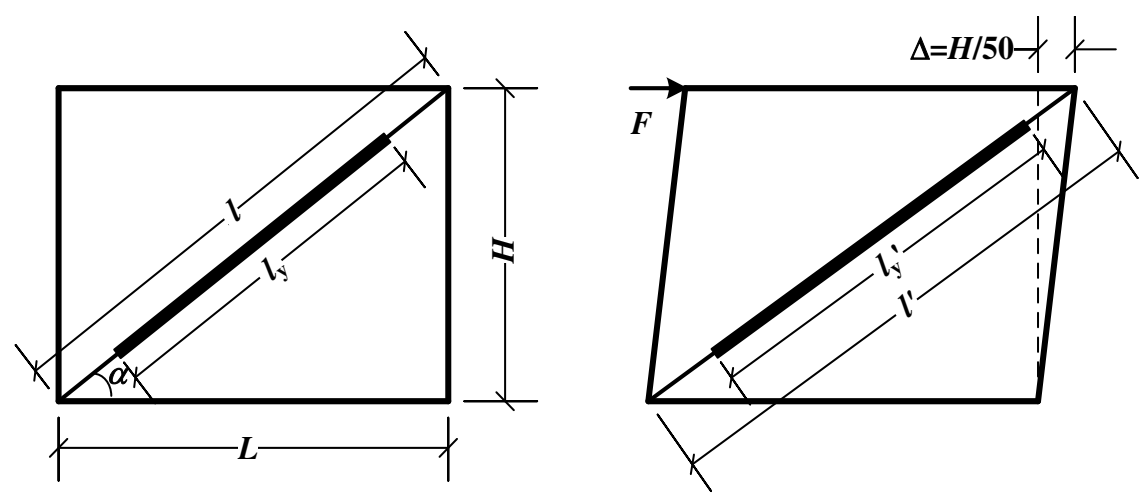

Fig. 3 Calculation of the maximum axial strain requirements

Prior to the standard loading process, the axial compressive load of half of the yield load

154 calculated from the FE model was preloaded to check whether the experimental devices and measuring instruments were reliable. After that, the standard loading process, in which three loading cycles with an axial strain of $0.25 \%, 0.50 \%, 0.75 \%, 1.00 \%, 1.50 \%$, and $2.00 \%$, were

157 performed successively to the specimens. The axial displacement loading protocol of the standard loading process for each specimen is tabulated in Table 2. According to this loading protocol, when the SAA-BRB specimens completed three $2.00 \%$ axial strain loadings, the cumulative plastic ductility would exceed the required ductility of 200 times of the yield strain as specified in AISC provisions (AISC 2016). The standard cyclic loading protocol of the axial strain with respect to the cycle number for each specimen is illustrated in Fig. 4.

Table 2 Axial displacement loading protocol

\begin{tabular}{cccc}
\hline Load step & Cycle number & Axial strain & Axial displacement $(\mathrm{mm})$ \\
\hline 1 & 3 & $\pm 0.25 \%$ & \pm 5.0 \\
2 & 3 & $\pm 0.50 \%$ & \pm 10.0 \\
3 & 3 & $\pm 0.75 \%$ & \pm 16.0 \\
4 & 3 & $\pm 1.00 \%$ & \pm 21.0 \\
5 & 3 & $\pm 1.50 \%$ & \pm 32.0 \\
6 & 3 & $\pm 2.00 \%$ & \pm 42.0 \\
\hline
\end{tabular}




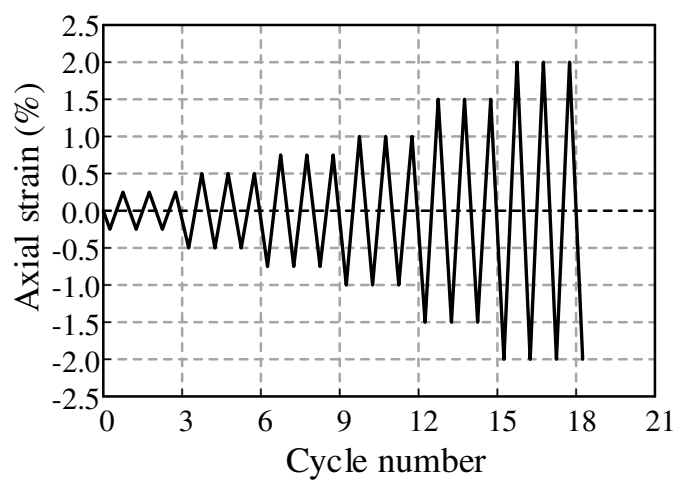

Fig. 4 Cyclic loading protocol

165

166

167

168

169

170

171

172

2.3 Measurement point arrangements

The measuring-point arrangements of strain gauges and displacement meters are presented in Fig. 5. The strain gauges were installed on the steel angles to record the axial strain. There were two measured sections: section 1 and section 2, and each section had eight measuring points. For displacement meters, in addition to two axial displacement meters (as shown in Fig. 2) used to examine the axial deformation, six lateral displacement meters were located near the mid-span bolt sets and at the quarter points of the restraining member along the longitudinal direction.

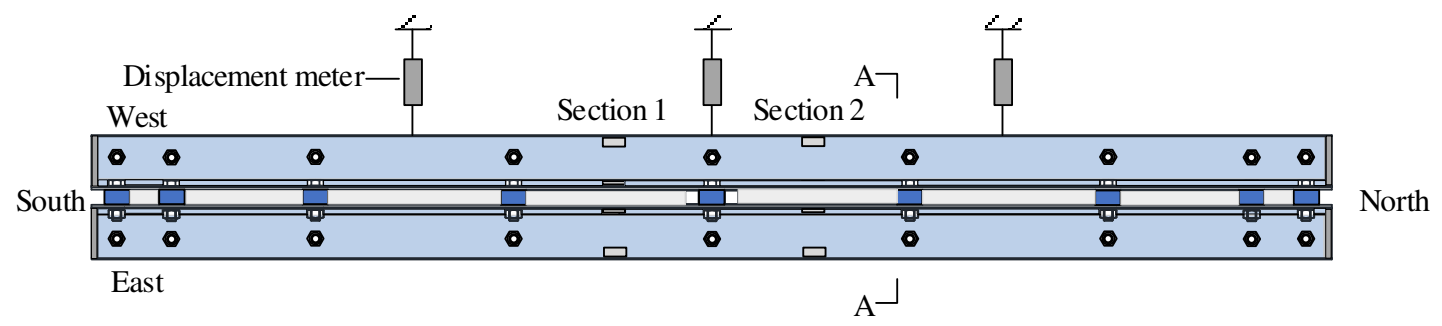

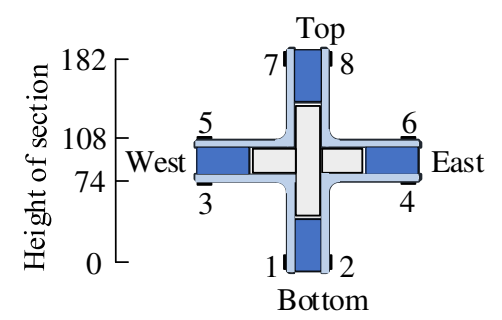

Sectional view (A-A) of S-1

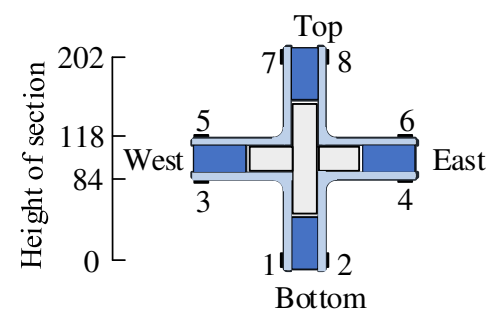

Sectional view (A-A) of S-2 or S-3

Fig. 5 Measuring-point arrangements 
176 maintained stable during the whole cyclic loading process, indicating that SAA-BRB has great

177 energy-dissipating capacity under severe seismic effects. Fig. 7 illustrates the deformation

178 condition corresponding to a core strain of $2.0 \%$. It is found that none of the specimens showed

179 global buckling or local buckling. This is because the restraining ratio of each specimen is

180 greater than the corresponding restraining ratio requirement.

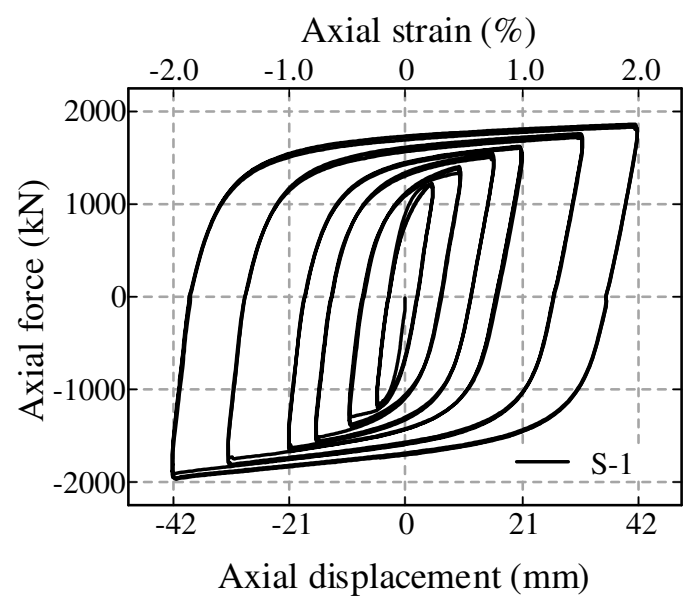

(a) Specimen S-1

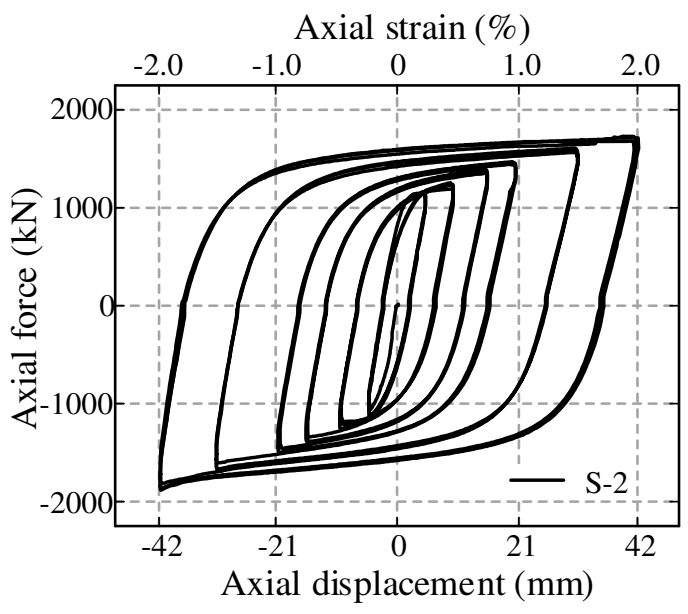

(b) Specimen S-2

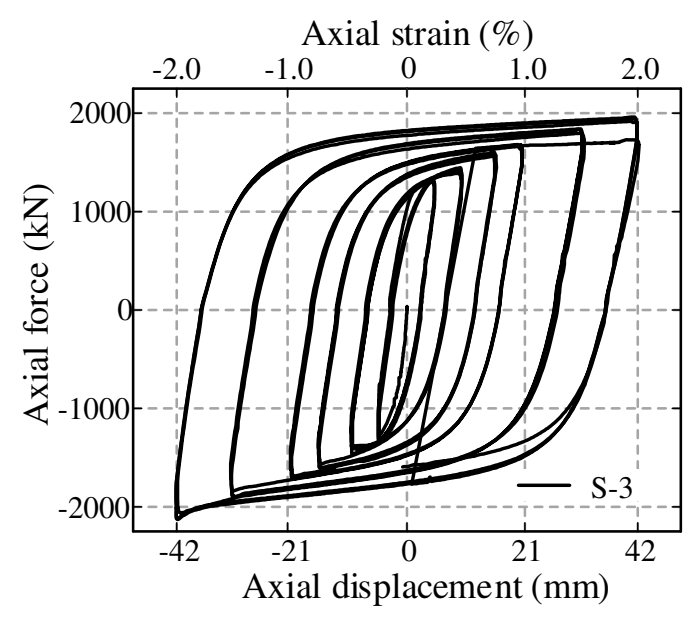

(c) Specimen S-3

Fig. 6 Hysteretic curves of specimens

181

182

183 


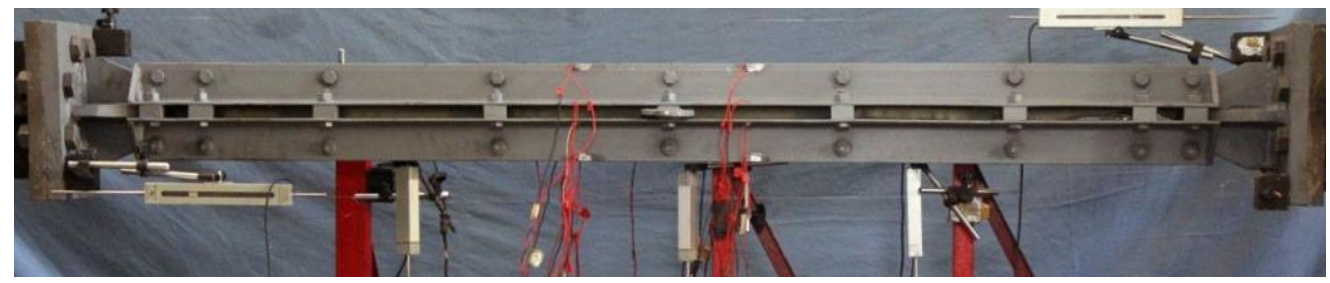

(a) Specimen S-1

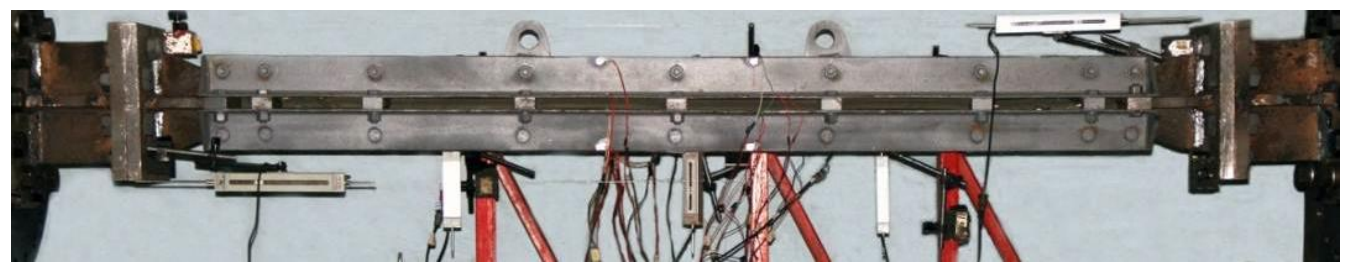

(b) Specimen S-2

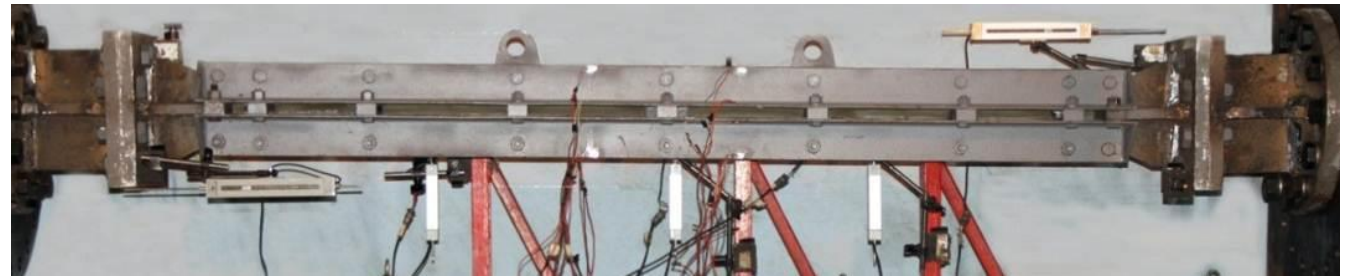

(c) Specimen S-3

Fig. 7 Deformation condition corresponding to $2.0 \%$ axial compression strain corresponding to the yield and peak points of all specimens are shown in Table 3 . When the

186 specimens reach $2.0 \%$ axial strain, the skeleton curves still maintain increasing trends, 187 indicating that the SAA-BRB specimens have a cyclic effect and great ductility under axial cyclic loading. Hence, it is conservative to take the peak point of the specimen as the last point of the skeleton curve to leave enough safety redundancy. In addition, each specimen curve has obvious inflection points, which are determined as the yield points of the specimen.

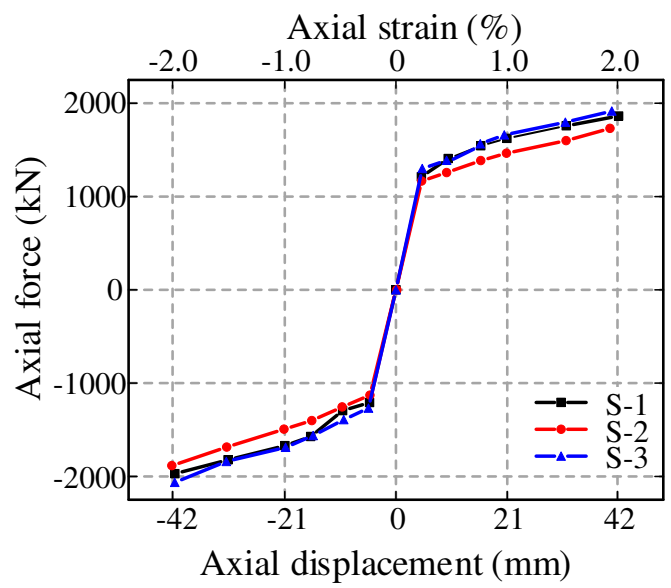

Fig. 8 Skeleton curves of specimens 
Table 3 Characteristic points and compression strength factors of specimens

\begin{tabular}{ccccccc}
\hline \multirow{2}{*}{ Specimen no. } & Loading direction & \multicolumn{2}{c}{ Yield points } & \multicolumn{2}{c}{ Peak points } & \multirow{2}{*}{$\beta$} \\
\cline { 3 - 6 } & & $F_{\mathrm{y}} / \mathrm{kN}$ & $\Delta_{\mathrm{y}} / \mathrm{mm}$ & $F_{\max } / \mathrm{kN}$ & $\Delta_{\max } / \mathrm{mm}$ & \\
\hline \multirow{2}{*}{$\mathrm{S}-1$} & Tension & 1212.58 & 4.77 & 1862.36 & 41.66 & \multirow{2}{*}{1.06} \\
& Compression & -1208.38 & -4.93 & -1971.56 & -41.33 & \\
\multirow{2}{*}{$\mathrm{S}-2$} & Tension & 1166.98 & 4.80 & 1730.37 & 40.04 & \multirow{2}{*}{1.09} \\
& Compression & -1129.18 & -4.85 & -1884.56 & -41.91 & \\
& Tension & 1299.57 & 4.92 & 1916.36 & 40.38 & \multirow{2}{*}{ S-3 } \\
& Compression & -1267.77 & -5.13 & -2063.96 & -41.36 & \\
\hline
\end{tabular}

2.4.2 Equivalent viscous damping ratio

193 Fig. 10 illustrates the equivalent viscous damping ratios of all specimens with respect to

194 the hysteretic loop. The energy-dissipating capacity of the specimen can be quantitatively

195 evaluated by the equivalent viscous damping ratio $\left(\xi_{\mathrm{eq}}\right)$, which can be calculated by Eq. (2). It

196 is seen that the equivalent viscous damping ratio of all specimens generally presents an

197 increasing trend with the increase of the hysteretic loops. Specifically, before the specimens

198 yielded (i.e., before the specimens reached $0.25 \%$ axial strain), the values of $\xi_{\text {eq }}$ ranged from

1990.2 to 0.3 . As the inner core gradually consumed energy during the loading process, the values

200 of $\xi_{\text {eq }}$ increased until they stabilized above 0.4 . Moreover, the maximum equivalent viscous

201 damping ratios of the three specimens all exceeded 0.5 , demonstrating that all the SAA-BRB

202 specimens exhibited excellent energy-dissipating capacity.

$$
\xi_{\mathrm{eq}}=\frac{1}{2 \pi} \cdot \frac{S_{(\mathrm{ABC}+\mathrm{CDA})}}{S_{(\mathrm{OBE}+\mathrm{ODF})}}
$$

203 in which $S_{(\mathrm{ABC}+\mathrm{CDA})}$ denotes the enveloping area of the hysteretic loop curve in Fig. 9; and

$204 S_{(\mathrm{OBE}+\mathrm{ODF})}$ denotes the area of the triangles OBE and ODF in Fig. 9.

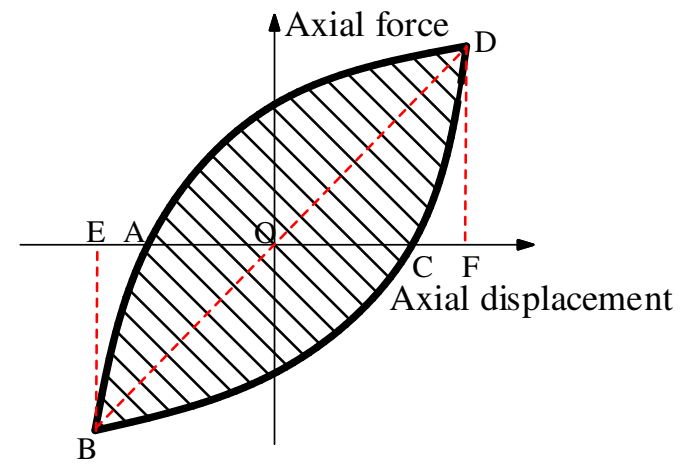

Fig. 9 Calculation of the equivalent viscous damping ratio 


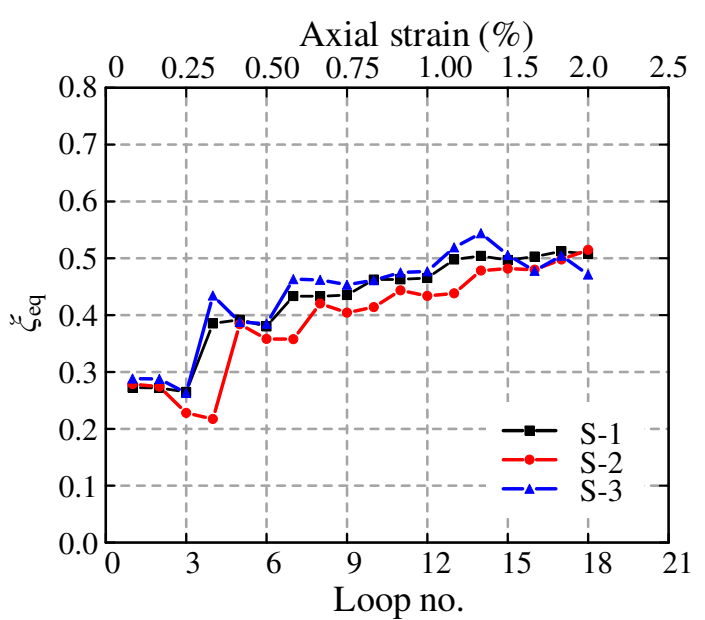

Fig. 10 Equivalent viscous damping ratio of specimens

206

207

208

\subsubsection{Compression strength adjustment factor}

The asymmetric response under tension and compression of the SAA-BRB can be evaluated by the compression strength adjustment factor $\beta$, which is defined by Eq. (3) (Jiang et al. 2017). The larger the $\beta$, the more obvious the asymmetric response is. It is calculated that the $\beta$ values of the three SAA-BRB specimens are 1.06, 1.09, and 1.08, respectively as tabulated in Table 3. ANSI/AISC 341-16 (AISC 2016) specified that the factor $\beta$ shall exceed unity but be no greater than 1.50. Therefore, it is shown that SAA-BRB has a reasonable hysteretic response under both tension and compression.

$$
\beta=\frac{P_{\mathrm{cmax}}^{i}}{P_{\mathrm{tmax}}^{i}}
$$

214 in which $P_{\mathrm{cmax}}^{i}$ and $P_{\mathrm{tmax}}^{i}$ are the loads corresponding to the compressive and tensile peak points

215 of the hysteretic curve, respectively.

\section{2.4.4 Stiffness degradation}

217 The stiffness degradation properties of all specimens are shown in Fig. 11. The axial

218 stiffness degradation curve can reflect the damage history of BRB under cyclic loads. 219 According to the Chinese code JGJ/T 101-2015 (JGJ 2015), the secant stiffness $K_{\mathrm{i}}$ is used to 220 express the stiffness of the specimen and its calculation is shown in Eq. (4). The horizontal 221 coordinate of Fig.11 is the displacement corresponding to the peak point of each loop in the compressive or tensile directions. It is found that the secant stiffnesses of all specimens are basically identical at the same load step. In addition, although the axial stiffness of three SAABRB specimens decreased during the loading process, the rate of stiffness degradation 
gradually slowed down. This shows that the SAA-BRB would not lose its stiffness

226 instantaneously during loading.

$$
K_{\mathrm{i}}=\frac{\left| \pm P_{\mathrm{i}}\right|}{| \pm \Delta|}
$$

227 in which $+P_{\mathrm{i}}$ and $-P_{\mathrm{i}}$ are the loads corresponding to tensile and compressive peak points of the 228 hysteretic curve at the $\mathrm{i}$-th cycle, respectively; $+\Delta_{\mathrm{i}}$ and $-\Delta_{\mathrm{i}}$ are the displacements corresponding to tensile and compressive peak points of the hysteretic curve at the i-th cycle, respectively.

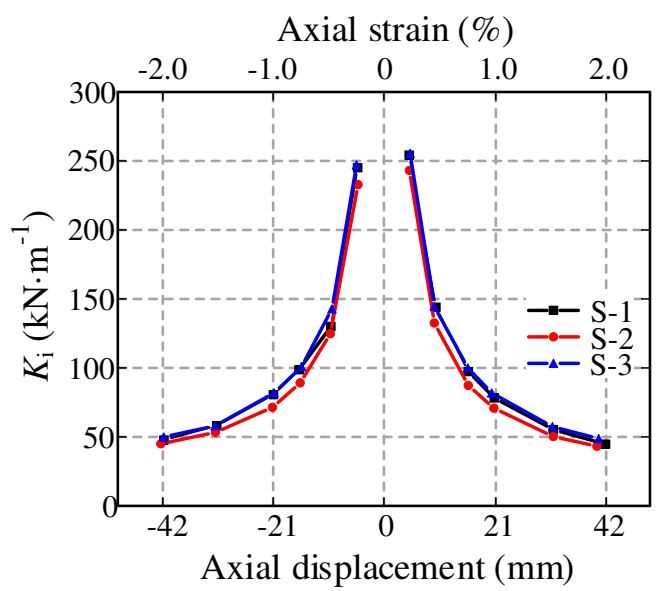

Fig. 11 Curves of axial secant stiffness degradation

\subsubsection{Longitudinal strain of restraining system}

231 Fig. 12 shows the longitudinal strain distribution and development of the external restraining system of the three specimens. Since the external restraining system was not loaded

233 when the inner core was under tension, the following analysis focuses on the longitudinal strain

234 of the external restraining member for the peak compressive displacement in each loading loop.

235 It is found that the difference between strain data of 1-1 section and 2-2 section is relatively 236 small, hence the data of 2-2 section is selected for strain analysis. Taking the average value of 237 a pair of strain data at the same height as the vertical coordinate and the height of the crosssection as the horizontal coordinate, the distribution of the longitudinal strains on the crosssection is plotted. It can be seen from Fig. 12 that the major bending direction of SAA-BRB

240 specimens is the up and down direction. Moreover, it is found that all strain values were less 241 than the yield strain (i.e., $1670 \mu \varepsilon$ for Q345 steel), indicating a good consistency with the stable 242 load-carrying capacities of these specimens during the loading process as shown in Fig. 7. 243 Theoretically, the strain distribution of the cross-section of the external restraining member is 
244 strictly linear if the restraining system can be regarded as a fully flexural member. However, 245 the sectional strain distribution of each SAA-BRB specimen depicted in Fig.12 shows that the 246 linearity is violated. This indicates that significant shear deformation exists in the restraining system and the plane-section assumption is inappropriate for the SAA-BRB. This is mainly caused by the discrete layout of the connecting bolts in the restraining system. When the inner core was under compression and developed multi-wave buckling mode, lateral thrust effect existed between the core and restraining system, thus causing friction along their interface. Hence, part of the axial load of the inner core was transmitted to the restraining system. This explains that the measured compressive strain is larger than tensile strain in the two measuringpoints which are symmetrical to the midline of the cross-section of each specimen.

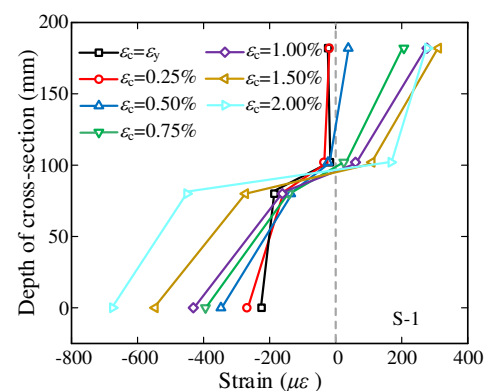

(a) Specimen S-1

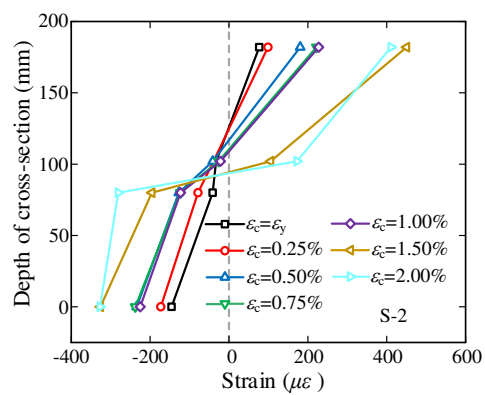

(b) Specimen S-2

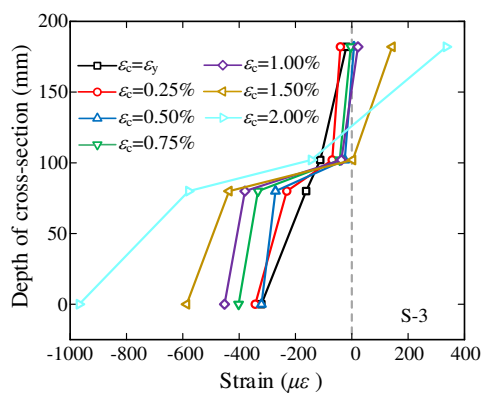

(c) Specimen S-3

Fig. 12 Distribution and development of longitudinal strains in restraining system

\section{FE model and validation}

3.1 Establishment of hysteretic model

In this section, the hysteretic behavior of the SAA-BRBs is simulated with ABAQUS by establishing a refined FE model. The refined FE model is depicted in Fig. 13. The four-node shell element (S4R) was used to simulate the steel angles, and the eight-node solid element (C3D8R) was used to simulate the inner core, spacer and high-strength bolts. It is pointed out that the high-strength bolts were set as infinitely rigid in the FE model because of their sufficient stiffness during the cyclic loading process of the experiments. Additionally, an air gap with a thickness of $1.0 \mathrm{~mm}$ on the surface of the inner core was set to ensure that the axial load transmission between the core and external restraining member was prevented. The specific geometric parameters of the FE model are identical to those of the specimens. 
considered. It is known that the material nonlinear constitutive relationship mainly consists of

269 yielding and strengthening criteria. In modelling the hysteretic behavior of SAA-BRBs, the von Mises yielding criterion was considered in the material, and a combined isotropic and

271 kinematic hardening model was selected as the strengthening criterion to consider the cyclic

272 effect shown in the experimental results. The yield stress values of the inner core and steel 273 angles were $288 \mathrm{MPa}$ and $380 \mathrm{MPa}$, respectively. It should be noted that these values were the same as the corresponding data tabulated in Table 1. Based on the suggestion of Guo et al.

275 (2017c), the parameters described below were well validated by the previous experiments for SAA-BRB. For isotropic hardening, a rate factor of 4.2 and a maximum change in yield stress of $\mathrm{Q}_{\infty}=150 \mathrm{MPa}$ were used in the model. Besides, the relevant moduli and rate factors for kinematic hardening were set as $\mathrm{C}_{1}=32 \mathrm{GPa}, \gamma_{1}=300 ; \mathrm{C}_{2}=6 \mathrm{GPa}, \gamma_{2}=150 ; \mathrm{C}_{3}=0.6 \mathrm{GPa}$, and $\gamma_{3}=22$.

280 For the contact behavior, the interface between the core and restraining system was simulated by hard contact along the normal direction and friction option along the tangent direction. The hard contact option allowed the interface to separate under tension but not penetrate under compression. The friction coefficient of 0.1 was adopted based on the suggestion of Chou and Chen (2010). In terms of the boundary conditions, both ends of SAABRB were modelled as fixed ends since the core was connected with the flange by highstrength bolts. Correspondingly, the rotational degree of freedom of the core at both ends was constrained. Additionally, an initial geometrical imperfection with a magnitude of $1 / 1000$ of the specimen length for the inner core was assigned to the FE model. The loading protocol was the same as that used in the experiment as listed in Table 2, except for the cycle number of each loading level, which was determined to be only one instead of three. 


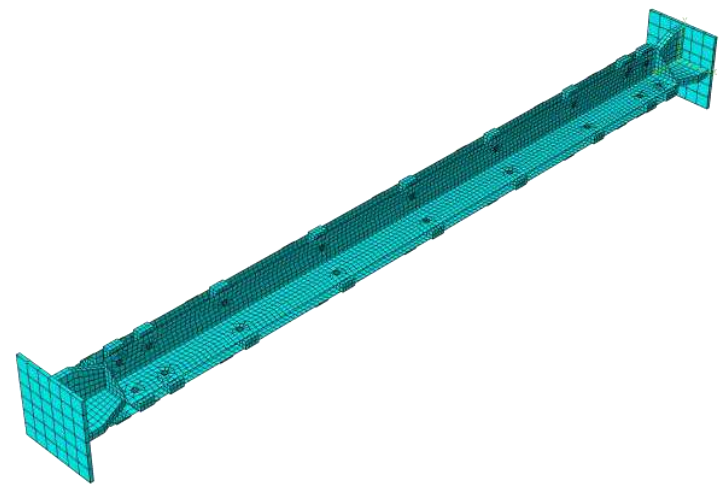

Fig. 13 FE model for hysteretic analysis

\subsection{Validation of FE model}

The comparison between the hysteretic curves obtained from FE simulations and experiments are illustrated in Fig. 14. It is seen that the hysteretic curves of the FE models and experiments can fit very well. Moreover, the model in the FE simulation showed the same stable load-carrying performance at the axial strain of $2.0 \%$ as the specimen in the experiment. Taking the model T-2 as an example, neither the restraining member nor the inner core was observed to undergo global buckling failure, while full cross-sectional yielding of inner core was exhibited at the axial strain of $2.0 \%$, as depicted in Fig. 15. Therefore, the refined FE model developed by software ABAQUS could precisely predict the hysteretic response of the SAABRB specimens under cyclic loads.

In addition, the unrestrained segment of the core remained elastic, while yielding had spread in the yield segment of the core. This further verifies the feasible design of unrestrained segment of the core by enlarging their cross-sectional size. Moreover, the maximum stress of the steel angles was far less than its yield stress of $380 \mathrm{MPa}$, indicating that the restraining system had sufficient flexural stiffness to limit the lateral deformation of the inner core. 


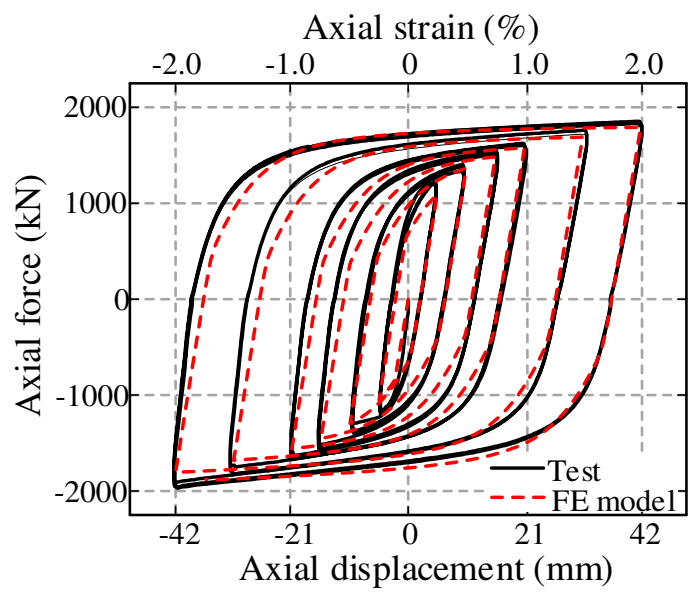

(a) Specimen S-1

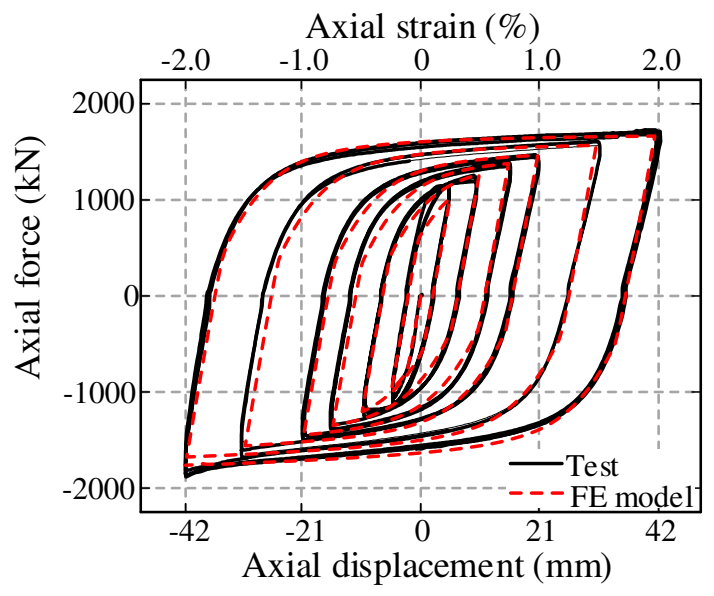

(b) Specimen S-2

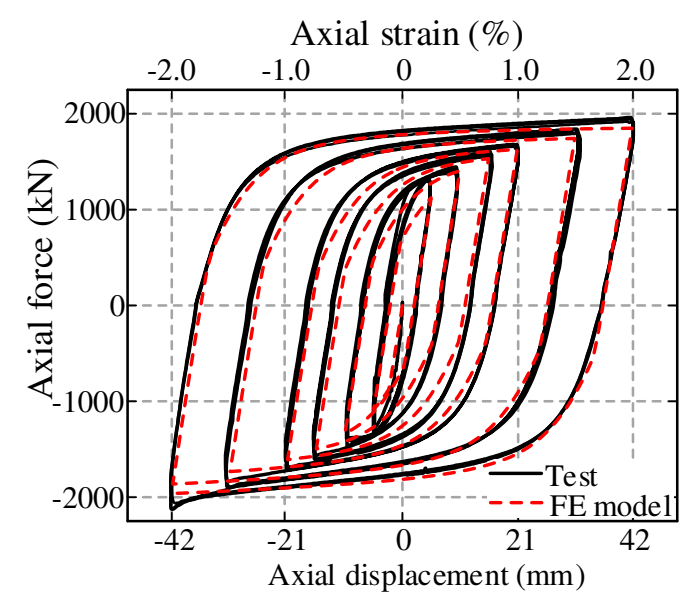

(c) Specimen S-3

Fig. 14 Comparison of experimental and numerical results of hysteretic curves

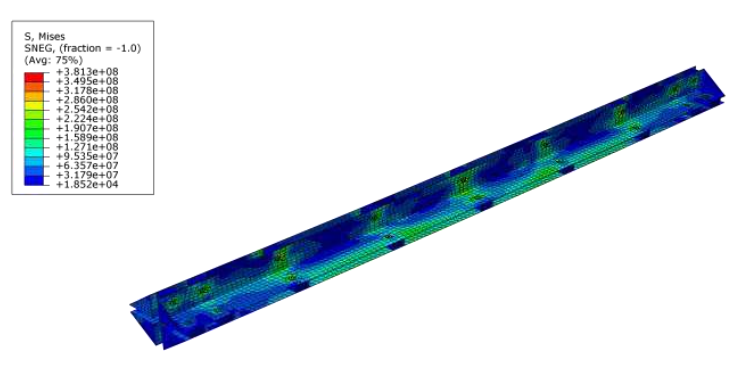

(a) Restraining member

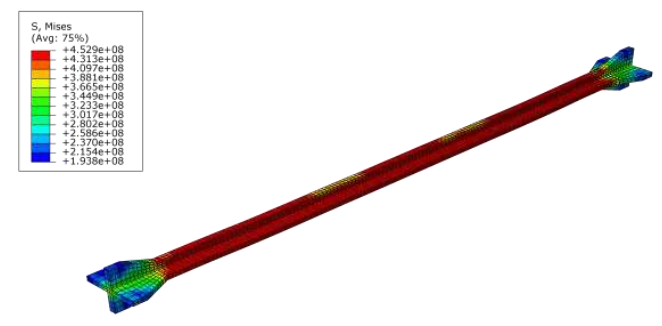

(b) Inner core

Fig. 15 Stress distribution of model T-2 at the axial strain of $2.0 \%$

\section{Theoretical analysis for global buckling design}

In this section, the formula for restraining ratio requirements of the SAA-BRB is derived.

310 In designing BRB, a restraining ratio $\zeta$ proposed by Fujimoto et al. (1988), reflecting the

311 restraining effect of the external restraining system on the inner core, is usually used to predict

312 the global buckling behavior and to examine the load-carrying performance of BRBs. It is 
313 known that the restraining ratio $\zeta$ is defined as the ratio of the Euler buckling load $P_{\mathrm{cr}}$ of BRB

314 to the yield load $P_{\mathrm{y}}$ of its inner core. The Euler buckling load $P_{\mathrm{cr}}$ of BRB can be considered as

315 the sum of the Euler buckling load $P_{\mathrm{cr}, \mathrm{c}}$ of inner core and the Euler buckling load $P_{\mathrm{cr}, \mathrm{r}}$ of

316 restraining system (Guo et al. 2016). Since the restraining system remains elastic while the

317 inner core yields during the loading process, leading to the negligible contribution of inner core

318 in calculating the Euler buckling load $P_{\text {cr }}$ of BRB. Therefore, the restraining ratio $\zeta$ is

319 commonly simplified as the ratio of the Euler buckling load $P_{\mathrm{cr}, \mathrm{r}}$ of the restraining system to

320 the yield load $P_{\mathrm{y}}$ of the inner core, as expressed by Eq. (5). Theoretically, as long as the

321 restraining ratio is slightly larger than unity, the cross-sectional strength of inner core can be

322 fully utilized, thus the BRB can maintain stable without global buckling during the whole

323 loading process. However, considering the influence of physical defects such as initial

324 geometric imperfection, residual stress, as well as the gap between the inner core and the

325 restraining system, the restraining ratio is required to be much larger than unity, indicating the

326 calculation of the requirements of the restraining ratio would be relatively complicated.

327 Through a reasonable design, the SAA-BRB will exhibit stable behavior instead of undergoing

328 global buckling before its inner steel core reaches the full cross-sectional yielding load (Tong

329 and Guo 2017). Although Tong and Guo (2018) have given the design suggestion about the

330 restraining requirement of SAA-BRB, there is still a lack of a precise design formula to

331 determine the restraining ratio as required. During the cyclic loading process, the external

332 restraining system can be regarded as a flexural member. Therefore, the formula of the critical

333 restraining ratio, i.e., the calculation of restraining ratio requirement of SAA-BRB, can be

334 derived by using the yielding criteria of outmost fiber for the restraining system section as the

335 elastic limit criterion.

$$
\zeta=\frac{P_{\mathrm{cr}, \mathrm{r}}}{P_{\mathrm{y}}}
$$

336 in which $P_{\text {cr,r }}$ is the Euler buckling load regarding the restraining system as an axial loading 337 member (Guo et al. 2015); $P_{\mathrm{y}}$ is the yield load of the inner core. 
For an ordinary BRB with an inner steel core encased by a uniform-sectional restraining member, the Euler buckling load of the restraining system can be calculated as

$$
P_{\mathrm{cr}, 0}=\frac{\pi^{2} E A_{0}}{\lambda_{0}^{2}}
$$

340 in which $E$ is the Young's modulus of the restraining member; $A_{0}$ is the cross-sectional area of

341 the restraining member and $\lambda_{0}$ is the slenderness ratio of the restraining member of ordinary

342 BRB, which can be calculated by Eq. (9).

343 It is known that the arrangement of discretely distributed bolts in the longitudinal direction

344 would result in a significant shear deformation of the restraining system, thus reducing the

345 Euler buckling load. Hence, a reduction factor $\omega$ is introduced to consider the effect of shear

346 deformation, as expressed in Eq. (7). The value of $\omega$ is closely related to the geometric

347 dimensions of the assembled BRB and is generally smaller than unity. For the SAA-BRB, Tong

348 and Guo (2018) introduced the derivation of the formula for $\omega$ through numerous FE

349 simulations and theoretical analysis, which is expressed by Eq. (8). It should be noted that the

350 derivation of reduction factor $\omega$ is based on the equivalence of the restraining system into a

351 four-chord battened column. In the equivalent relation, the four angle steels were equivalent to

352 four chord members, and the spacers were equivalent to batten plates (Tong and Guo 2018).

$$
\begin{gathered}
P_{\mathrm{cr}, \mathrm{r}}=\omega P_{\mathrm{cr}, 0} \\
\omega=\left[1+\frac{\pi^{2} \lambda_{1}^{2}}{12 \lambda_{0}^{2}}+17.4 \frac{\lambda_{1}^{0.55}}{\lambda_{0}^{1.27}}\right]^{-1}
\end{gathered}
$$

in which $\lambda_{1}$ is the slenderness ratio of a single chord member between adjacent batten plates.

$$
\lambda_{0}=\frac{l_{0}}{\sqrt{I_{0} / A_{0}}}
$$

354 in which $\lambda_{0}$ is the slenderness ratio regarding the external restraining system as a member with

355 a uniform cross-section; $l_{0}$ is the length of the external restraining system; $I_{0}$ is the moment of

356 inertia regarding the external restraining system as a whole about the $x-x$ axis and $A_{0}$ is the

357 cross-sectional area of the external restraining system. 


$$
\lambda_{1}=\frac{l_{1}}{\sqrt{I_{1} / A_{1}}}
$$

358 in which $l_{1}$ is the longitudinal spacing between adjacent high-strength bolts, which is equal to

359 the spacing between adjacent batten plates; $I_{1}$ is the moment of inertia of a single angle about

360 the $x_{1}-x_{1}$ axis, $A_{1}$ is the cross-sectional area of a single steel angle. Besides, the $x-x$ axis for

361 calculating the $I_{0}$ and $x_{1}-x_{1}$ axis for calculating the $I_{1}$ are shown in Fig. 16.

362

363

364

365

366

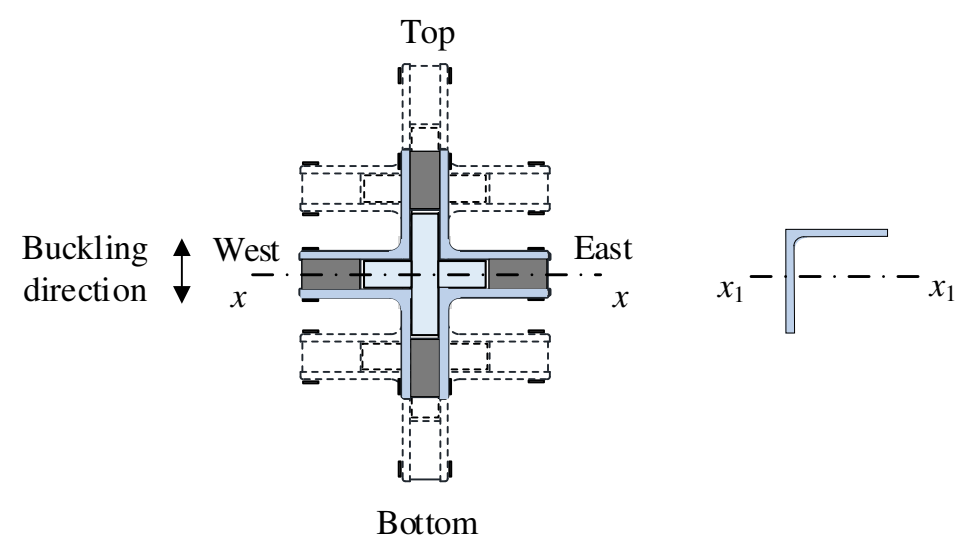

Fig. 16 Axis of $x-x$ and $x_{1}-x_{1}$ for calculation

When the load reaches the value corresponding to the yield load of the inner steel core, the maximum lateral deformation $v_{\max }$ at the mid-span of the restraining system in the longitudinal direction can be expressed as (Timoshenko et al. 1962)

$$
v_{\max }=\frac{v_{0}+g}{1-\frac{P_{\mathrm{y}}}{P_{\mathrm{cr}, \mathrm{r}}}}
$$

367 in which $v_{0}(=L / 1000)$ is the amplitude of initial imperfection and $g$ is the gap between the inner 368 steel core and the restraining member.

369 When global buckling occurs, the maximum bending moment $M_{\max }$ at the mid-span of the 370 restraining system can be expressed as

$$
M_{\max }=P_{\mathrm{y}} v_{\max }
$$

371 Correspondingly, the maximum stress of the restraining system can be calculated by Eq.

372 (13). It should be aware that the plastic section modulus $W$ is multiplied by the reduction factor $373 \omega$ to express the weakening effect of discrete bolt connection. 


$$
\sigma_{\max }=\frac{M_{\max }}{\omega W}
$$

Based on the yielding criteria of outmost fiber for the restraining system section, the overall stability requirement of the SAA-BRB subjected to axial compressive load can be expressed as

$$
\sigma_{\max } \leq f_{\text {yr }}
$$

in which $f_{\mathrm{y}, \mathrm{r}}$ is the yield stress of the restraining system.

By combining Eqs. (5), (7), and (11) - (14), a calculation for a lower limit of restraining ratio [ל] for SAA-BRB can be expressed as Eq. (15), which is used for global buckling design of SAA-BRB. For convenience of the design by engineers, $\zeta /[\zeta]$ can be determined as a design coefficient. When the coefficient $\zeta /[\zeta]$ value exceeds unity, i.e. $\zeta>[\zeta]$, the satisfactory design of the SAA-BRB can be achieved by preventing its global buckling.

$$
\zeta \geq \frac{\omega W f_{\mathrm{yr}}}{\omega W f_{\mathrm{yr}}-P_{\mathrm{y}}\left(v_{0}+g\right)}=[\zeta]
$$

\section{Parametric study and design formula verification}

Based on the proposed FE model, 24 FE hysteretic models listed in Table 4 were analyzed to conduct a parametric study and to verify the accuracy of the proposed formula (Eq. (15)) for global buckling design of SAA-BRB. The geometric dimensions are listed in Table 4, and the geometric parameters and material properties of the models are identical with the specimens in the experimental program. These 24 models were divided into 3 groups, and the core sectional area of models in each group were identical, and their corresponding values were $4356 \mathrm{~mm}^{2}$, $4796 \mathrm{~mm}^{2}$, and $6566 \mathrm{~mm}^{2}$, respectively. Besides, the loading protocol in the FE extensive analysis was determined according to the standard loading stage shown in Fig. 4, yet each loading step was applied for only one cycle.

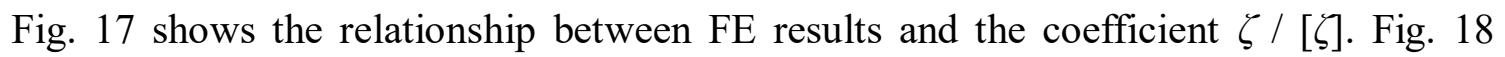
presents the hysteretic curves of representative FE models. The solid rectangles in Fig.17 represent that the models maintained stable while the hollow rectangles corresponded to models subjected to global buckling failure. When the $\zeta /[\zeta]$ value is greater than unity, the 
397 global buckling failure of the FE model would be prevented and the corresponding hysteretic 398 curves of the models maintained stable; when the $\zeta /[\zeta]$ value is less than unity, the global 399 buckling mode would occur in the FE model. Overall, a critical value (i.e., the value of red line) 400 exists in Fig. 17, below which a stable bearing-capacity would transition to a global buckling 401 mode. Taking model M-3 as an example, its $\zeta /[\zeta]$ value is 1.40. It is seen from Fig. 18(a) that 402 its hysteretic curve is full and symmetrical, indicating a stable bearing-capacity and satisfactory 403 energy-dissipation capability during the whole loading process. Moreover, it is observed from 404 Fig. 19 that the yielding had spread in the core while the restraining system remained elastic. 405 This indicates that the full-sectional yielding load of the inner core could be achieved before 406 reaching 2.0\% axial strain. Furthermore, taking model M-13 as an example, its $\zeta /[\zeta]$ value is 407 0.81. It can be seen from Fig. 20 that the model M-13 exhibited global buckling with a single408 wave lateral deformation at the load step of $1.5 \%$ axial strain, thus severely pinched hysteretic 409 curve was obtained as illustrated in Fig. 18(e). Besides, stress concentrations were observed at 410 the mid-span and both ends of the yield segment of the core, indicating that full cross-sectional 411 yield load of the inner core could not be reached. Therefore, the accuracy of the formula (Eq. 412 (15)) of restraining ratio requirements for global buckling design of SAA-BRBs is validated, 413 which can provide a valuable reference for practical designs. 
Table 4 Information of hysteretic models and FE results

\begin{tabular}{|c|c|c|c|c|c|c|c|c|c|c|c|}
\hline \multirow{2}{*}{ Group no. } & \multirow{2}{*}{ Hysteretic model no. } & \multicolumn{5}{|c|}{ Geometrical dimensions } & \multirow{2}{*}{$\omega$} & \multirow{2}{*}{$\zeta$} & \multirow{2}{*}[\zeta]{} & \multirow{2}{*}{$\zeta /[\zeta]$} & \multirow{2}{*}{ FE results ${ }^{\mathrm{a}}$} \\
\hline & & $l(\mathrm{~mm})$ & $l_{\mathrm{y}}(\mathrm{mm})$ & $b \times t(\mathrm{~mm})$ & $b_{\mathrm{a}} \times t_{\mathrm{a}}(\mathrm{mm})$ & $A_{\mathrm{c}}\left(\mathrm{mm}^{2}\right)$ & & & & & \\
\hline \multirow{8}{*}{1} & M-1 & 1700 & 1400 & $110 \times 22$ & $100 \times 6$ & \multirow{8}{*}{4356} & 0.548 & 5.32 & 1.99 & 2.68 & $\mathrm{~S}$ \\
\hline & M-2 & 2000 & 1700 & $110 \times 22$ & $100 \times 6$ & & 0.580 & 3.82 & 2.02 & 1.89 & $\mathrm{~S}$ \\
\hline & M-3 & 2300 & 2000 & $110 \times 22$ & $100 \times 6$ & & 0.606 & 2.88 & 2.06 & 1.40 & $\mathrm{~S}$ \\
\hline & M-4 & 2600 & 2300 & $110 \times 22$ & $100 \times 6$ & & 0.627 & 2.26 & 2.10 & 1.07 & $\mathrm{~S}$ \\
\hline & M-5 & 2900 & 2600 & $110 \times 22$ & $100 \times 6$ & & 0.645 & 1.82 & 2.15 & 0.85 & GB \\
\hline & M-6 & 3200 & 2900 & $110 \times 22$ & $100 \times 6$ & & 0.661 & 1.50 & 2.20 & 0.68 & GB \\
\hline & M-7 & 3500 & 3200 & $110 \times 22$ & $100 \times 6$ & & 0.675 & 1.25 & 2.25 & 0.56 & GB \\
\hline & M-8 & 3800 & 3500 & $110 \times 22$ & $100 \times 6$ & & 0.687 & 1.07 & 2.30 & 0.46 & GB \\
\hline \multirow{8}{*}{2} & M-9 & 2300 & 2000 & $120 \times 22$ & $110 \times 7$ & \multirow{8}{*}{4796} & 0.595 & 3.86 & 1.94 & 1.99 & $\mathrm{~S}$ \\
\hline & M-10 & 2600 & 2300 & $120 \times 22$ & $110 \times 7$ & & 0.617 & 3.03 & 1.97 & 1.53 & $\mathrm{~S}$ \\
\hline & M-11 & 2900 & 2600 & $120 \times 22$ & $110 \times 7$ & & 0.636 & 2.44 & 2.01 & 1.22 & $\mathrm{~S}$ \\
\hline & M-12 & 3200 & 2900 & $120 \times 22$ & $110 \times 7$ & & 0.652 & 2.01 & 2.04 & 0.99 & GB \\
\hline & M-13 & 3500 & 3200 & $120 \times 22$ & $110 \times 7$ & & 0.666 & 1.69 & 2.07 & 0.81 & GB \\
\hline & M-14 & 3800 & 3500 & $120 \times 22$ & $110 \times 7$ & & 0.679 & 1.44 & 2.11 & 0.68 & GB \\
\hline & M-15 & 4100 & 3800 & $120 \times 22$ & $110 \times 7$ & & 0.690 & 1.24 & 2.15 & 0.58 & GB \\
\hline & M-16 & 4400 & 4100 & $120 \times 22$ & $110 \times 7$ & & 0.700 & 1.08 & 2.18 & 0.49 & GB \\
\hline \multirow{8}{*}{3} & M-17 & 2300 & 2000 & $120 \times 22$ & $120 \times 8$ & \multirow{8}{*}{6556} & 0.585 & 4.01 & 1.96 & 2.04 & $\mathrm{~S}$ \\
\hline & M-18 & 2600 & 2300 & $160 \times 22$ & $120 \times 8$ & & 0.607 & 3.15 & 2.00 & 1.58 & $\mathrm{~S}$ \\
\hline & M-19 & 2900 & 2600 & $160 \times 22$ & $120 \times 8$ & & 0.626 & 2.54 & 2.03 & 1.25 & $\mathrm{~S}$ \\
\hline & M-20 & 3200 & 2900 & $160 \times 22$ & $120 \times 8$ & & 0.643 & 2.10 & 2.06 & 1.02 & $\mathrm{~S}$ \\
\hline & M-21 & 3500 & 3200 & $160 \times 22$ & $120 \times 8$ & & 0.657 & 1.76 & 2.10 & 0.84 & GB \\
\hline & M-22 & 3800 & 3500 & $160 \times 22$ & $120 \times 8$ & & 0.670 & 1.50 & 2.14 & 0.70 & GB \\
\hline & M-23 & 4100 & 3800 & $160 \times 22$ & $120 \times 8$ & & 0.682 & 1.29 & 2.18 & 0.59 & GB \\
\hline & M-24 & 4400 & 4100 & $160 \times 22$ & $120 \times 8$ & & 0.692 & 1.13 & 2.22 & 0.51 & GB \\
\hline
\end{tabular}

415 Notes: FE results a represents the failure mode of SAA-BRBs, in which "S” represents "stable"; "GB” represents “global buckling”. 


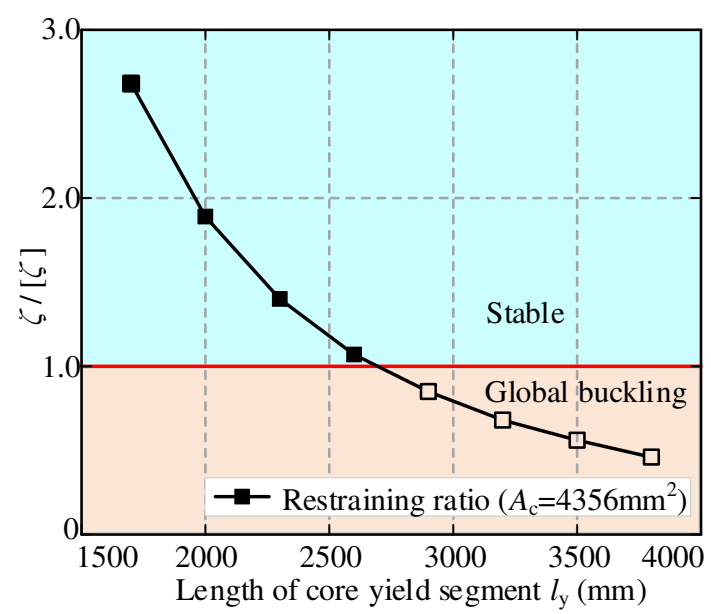

(a) Group $1\left(A_{\mathrm{c}}=4356 \mathrm{~mm}^{2}\right)$

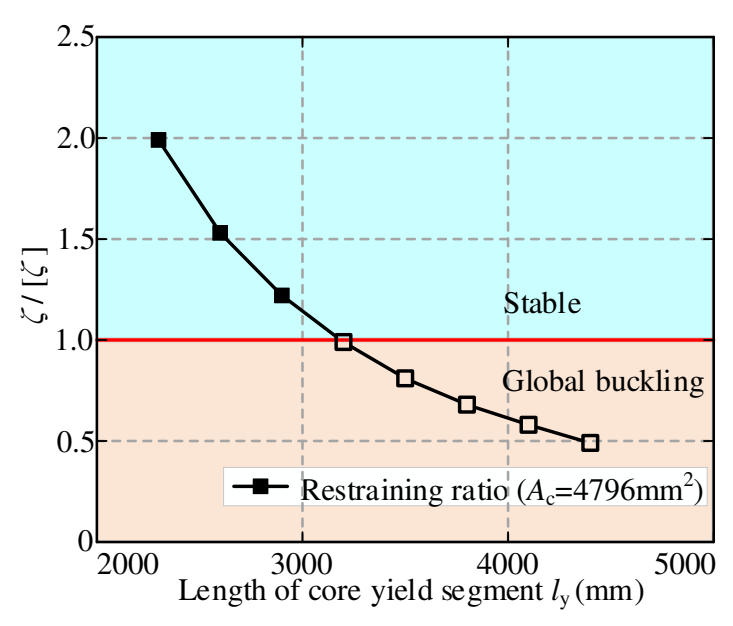

(b) Group $2\left(A_{\mathrm{c}}=4796 \mathrm{~mm}^{2}\right)$

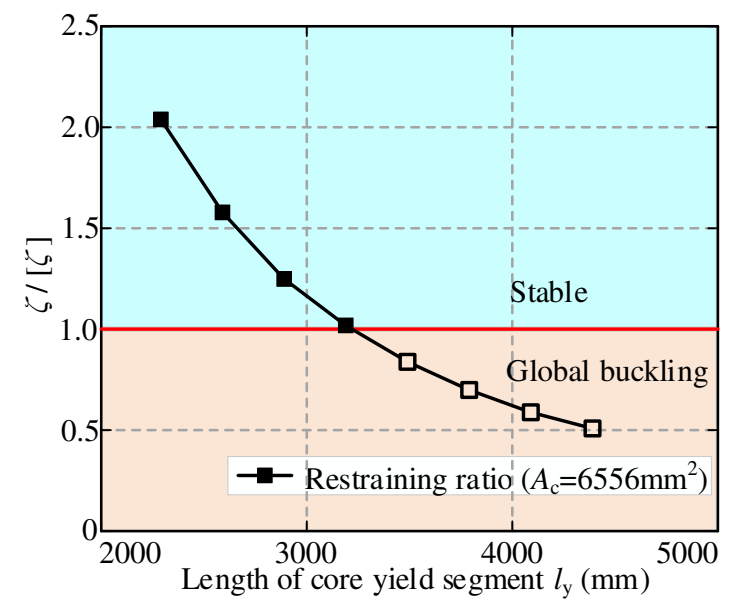

(c) Group $3\left(A_{\mathrm{c}}=6556 \mathrm{~mm}^{2}\right)$

Fig. 17 Verification of restraining ratio requirements

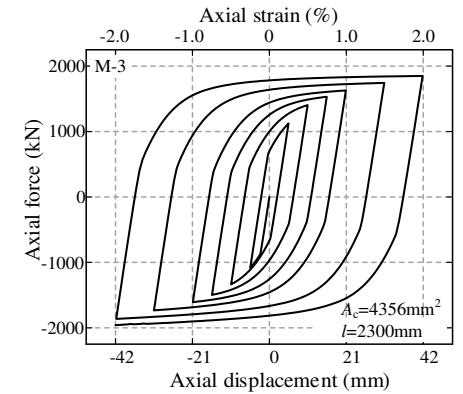

(a) $\mathrm{M}-3(\zeta=2.88,[\zeta]=2.06)$

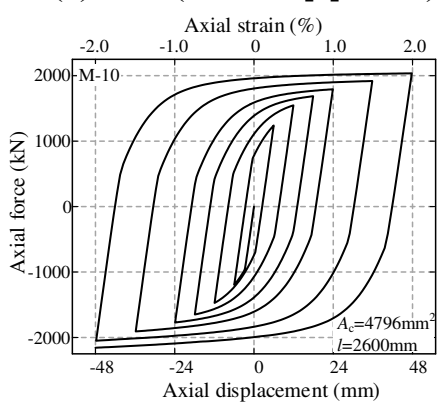

(d) $\mathrm{M}-10(\zeta=2.44,[\zeta]=2.01)$

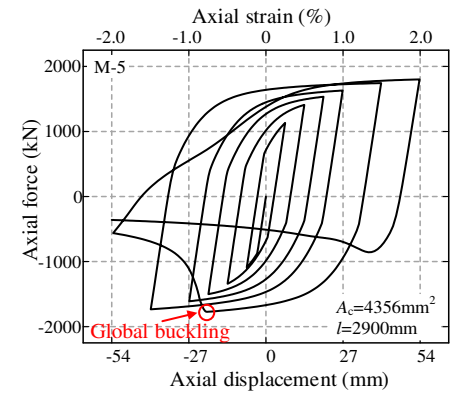

(b) $\mathrm{M}-5(\zeta=1.82,[\zeta]=2.15)$

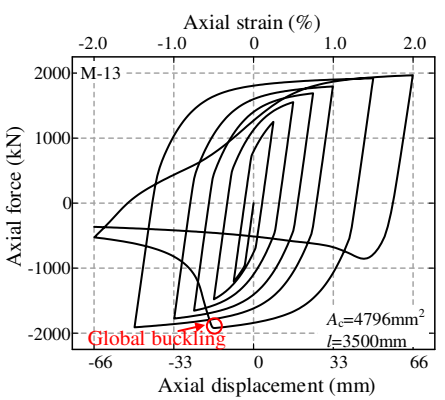

(e) $\mathrm{M}-13(\zeta=1.69,[\zeta]=2.07)$

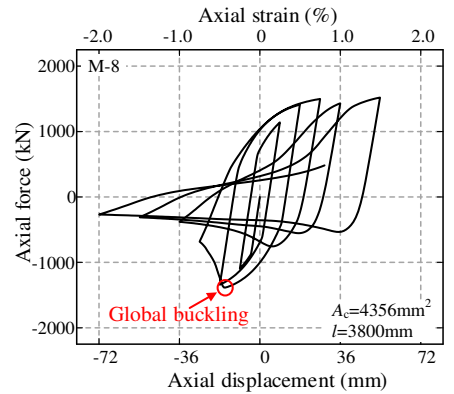

(c) $\mathrm{M}-8(\zeta=1.07,[\zeta]=2.30)$

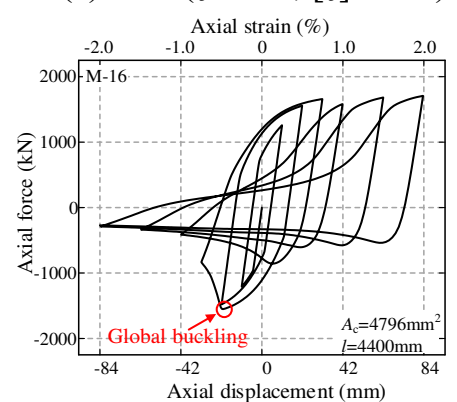

(f) $\mathrm{M}-16(\zeta=1.08,[\zeta]=2.18)$ 


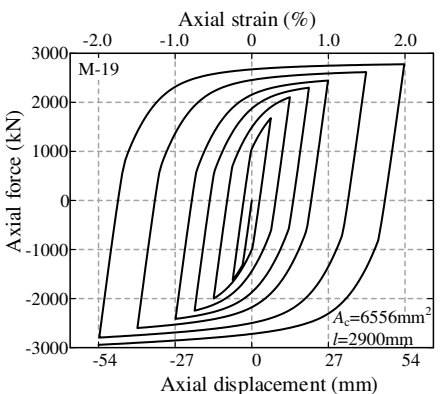

(g) $\mathrm{M}-19(\zeta=2.54,[\zeta]=2.03)$

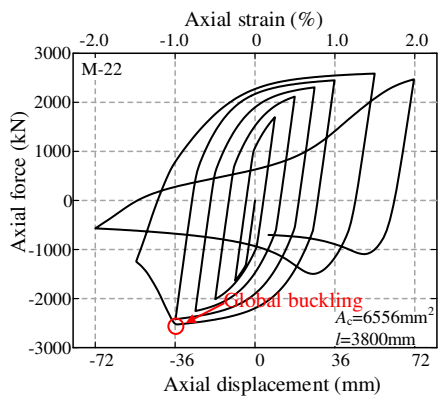

(h) $\mathrm{M}-22(\zeta=2.54,[\zeta]=2.03)$

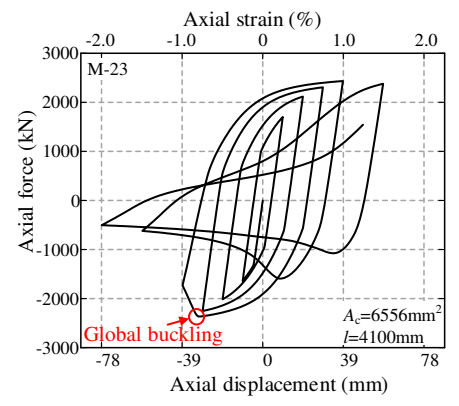

(i) $\mathrm{M}-23(\zeta=1.29,[\zeta]=2.18)$

Fig. 18 Hysteretic curves of FE models

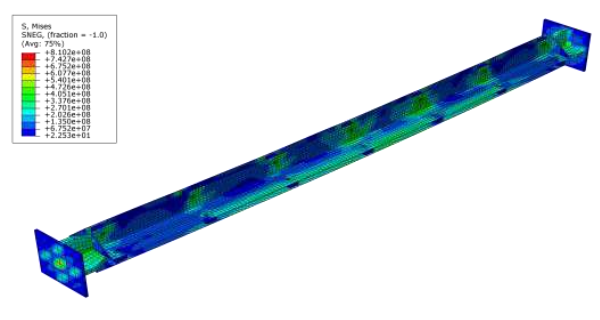

(a) Whole member

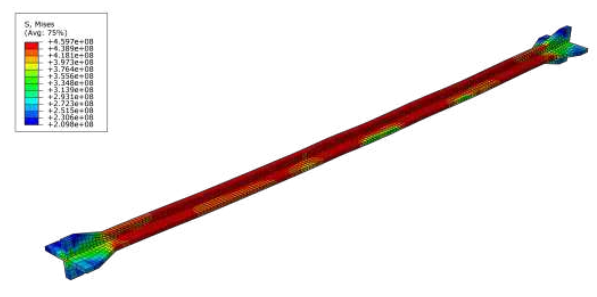

(b) Inner core

Fig. 19 Stress distribution of the SAA-BRB (model M-3)

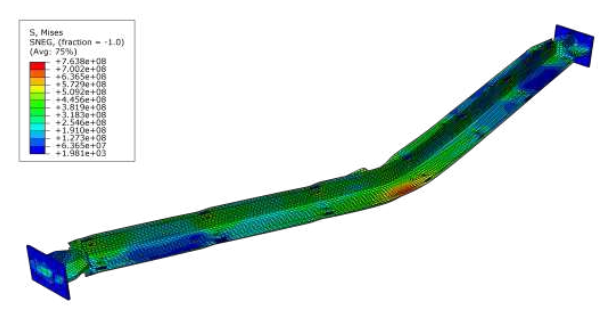

(a) Whole member

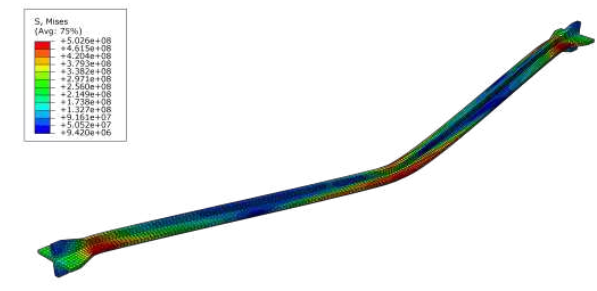

(b) Inner core

Fig. 20 Stress distribution of the SAA-BRB (model M-13)

\section{Conclusions}

In this study, the hysteretic responses of steel-angles-assembled buckling-restrained brace (SAA-BRB) were investigated experimentally and numerically. A design formula of restraining ratio requirements with high accuracy for predicting the global buckling of SAA-BRB was proposed and verified by parametric FE hysteretic analysis. The following conclusions can be drawn:

(1) Through the experiments conducted on three specimens, the hysteretic curves and skeleton curves were obtained. It is found from the experimental results that all specimens maintained stable without global buckling, indicating that a proper-designed SAA-BRB can exhibit satisfactory hysteretic performance under cyclic loads. Based on the analysis of results from the experiments, it is found that the maximum equivalent viscous damping ratios of all 
427 specimens are greater than 0.5 and the compression strength adjustment factors are lower than 4281.50 as specified in AISC. This shows that the SAA-BRB has favorable load-carrying 429 performance and energy-dissipating capacity. In addition, the plane-section assumption is 430 violated for SAA-BRB by analyzing the longitudinal strain distribution of the retraining system. 431 (2) A refined FE model considering geometric nonlinearity and material nonlinearity was 432 established. It is demonstrated that the results from FE analysis were consistent with those 433 obtained from experiments, indicating the validity of the established model in predicting the 434 hysteretic responses of SAA-BRB.

435 (3) Based on the yielding criteria of outmost fiber for the restraining system section, the 436 theoretical calculation formula of restraining ratio requirements [ $\zeta$ ] is derived. A parametric 437 analysis involving 24 FE models was performed to investigate the accuracy of the proposed 438 formula. It is verified that the proposed formula of restraining ratio requirement $[\zeta]$ is suitable 439 for global buckling design of SAA-BRB in engineering practice. 


\section{References}

441 AISC (2016) Seismic provisions for structural steel buildings. AISC, Chicago

442 Ariyaratana C, Fahnestock LA (2011) Evaluation of buckling-restrained braced frame seismic performance considering reserve strength. Eng Struct 33(1):77-89. https://doi.org/10.1016/j.engstruct.2010.09.020

Black CJ, Makris N, Aiken ID (2004) Component testing, seismic evaluation and characterization of BucklingRestrained braces. Journal of structural engineering (New York, N.Y.) 130(6):880-894. https://doi.org/10.1061/(ASCE)0733-9445(2004)130:6(880)

Chou C, Chen S (2010) Subassemblage tests and finite element analyses of sandwiched buckling-restrained braces. Eng Struct 32(8):2108-2121. https://doi.org/10.1016/j.engstruct.2010.03.014

Chou C, Hsiao C, Chen Z, Chung P, Pham D (2019) Seismic loading tests of full-scale two-story steel building frames with self-centering braces and buckling-restrained braces. Thin Wall Struct 140:168-181. https://doi.org/10.1016/j.tws.2019.03.024

Dehghani M, Tremblay R (2018) Design and full-scale experimental evaluation of a seismically endurant steel buckling-restrained brace system. Earthq Eng Struct D 47(1):105-129. https://doi.org/10.1002/eqe.2941

Ding YK (2014) Cyclic tests for unbonded steel plate brace encased in reinforced concrete panel or light-weight assembled steel panel. J Constr Steel Res 94:91-102. https://doi.org/10.1016/j.jcsr.2013.11.009

Eryasar ME, Topkaya C (2010) An experimental study on steel-encased buckling-restrained brace hysteretic dampers. Earthq Eng Struct D 39(5):561-581. https://doi.org/10.1002/eqe.959

Fujimoto M (1988) A study on the unbonded brace encasted in bucking-restrained concrete and steel tube. Journal of Str. Engineering, AIJ 34

461

Fujimoto M, Wada A, Saeki E, Takeuchi T, Watanabe A (1990) Development of unbonded brace. Quarterly Column 115(1):91-96

463

GB (2010) Code for seismic design of buildings. China Plan Publishing Company, Beijing

GB (2017) Code for design of steel structures. China Plan Publishing Company, Beijing

Guo Y, Fu P, Zhou P, Tong J (2016) Elastic buckling and load resistance of a single cross-arm pre-tensioned cable stayed buckling-restrained brace. Eng Struct 126:516-530

Guo Y, Zhang B, Zhu B, Zhou P, Zhang Y, Tong J (2017a) Theoretical and experimental studies of battened buckling-restrained braces. Eng Struct 136:312-328. https://doi.org/10.1016/j.engstruct.2017.01.034

Guo Y, Tong J, Zhang B, Zhu B, Pi Y (2017b) Theoretical and experimental investigation of core-separated buckling-restrained braces. J Constr Steel Res 135:137-149. https://doi.org/10.1016/j.jcsr.2017.04.019

Guo Y, Tong J, Wang X, Zhang B (2017c) Subassemblage tests and numerical analyses of buckling-restrained braces under pre-compression. Eng Struct 138:473-489. https://doi.org/10.1016/j.engstruct.2017.02.046

Guo Y, Tong J, Wang X, Zhou P (2018) Subassemblage tests and design of steel channels assembled bucklingrestrained braces. B Earthq Eng 16(9):4191-4224. https://doi.org/10.1007/s10518-018-0337-5

Guo Y, Zhang B, Jiang Z, Chen H (2015) Critical load and application of core-separated buckling-restrained braces. J Constr Steel Res 106:1-10. https://doi.org/10.1016/j.jcsr.2014.11.011

Guo Y, Zhou P, Andrew Bradford M, Pi Y, Tong J, Fu P (2017) Theoretical and numerical studies of elastic buckling and load resistance of double cross-arm pre-tensioned cable stayed buckling-restrained braces. Eng Struct 153:674-699. https://doi.org/10.1016/j.engstruct.2017.10.064

Hosseinzadeh S, Mohebi B (2016) Seismic evaluation of all-steel buckling restrained braces using finite element analysis. J Constr Steel Res 119:76-84. https://doi.org/10.1016/j.jcsr.2015.12.014

JGJ (2015) Specification for seismic test of building. China Plan Publishing Company, Beijing 
Jia L, Ge H, Maruyama R, Shinohara K (2017) Development of a novel high-performance all-steel fish-bone shaped buckling-restrained brace. Eng Struct 138:105-119. https://doi.org/10.1016/j.engstruct.2017.02.006

Jiang ZQ, Guo YL, Zhang AL, Dou C, Zhang CX (2017) Experimental study of the pinned double rectangular tube assembled buckling-restrained brace. J Zhejiang Univ-Sc a 18(1):20-32. https://doi.org/10.1631/jzus.A1600483

Ju YK, Kim M, Kim J, Kim S (2009) Component tests of buckling-restrained braces with unconstrained length. Eng Struct 31(2):507-516. https://doi.org/10.1016/j.engstruct.2008.09.014

Judd JP, Marinovic I, Eatherton MR, Hyder C, Phillips AR, Tola AT, Charney FA (2016) Cyclic tests of all-steel web-restrained buckling-restrained brace subassemblages. J Constr Steel Res 125:164-172. https://doi.org/10.1016/j.jcsr.2016.06.007

Kiggins S, Uang C (2006) Reducing residual drift of buckling-restrained braced frames as a dual system. Eng Struct 28(11):1525-1532. https://doi.org/10.1016/j.engstruct.2005.10.023

Metelli G, Bregoli G, Genna F (2016) Experimental study on the lateral thrust generated by core buckling in bolted-BRBs. J Constr Steel Res 122:409-420. https://doi.org/10.1016/j.jcsr.2016.04.004

Mirtaheri SM, Nazeryan M, Bahrani MK, Nooralizadeh A, Montazerian L, Naserifard M (2017) Local and global buckling condition of all-steel buckling restrained braces. Steel Compos Struct 23(2):217-228. https://doi.org/https://doi.org/10.12989/scs.2017.23.2.217

Mochizuki S, Murata Y, Andou N, Takahashi S (1979) Experimental study on buckling of unbonded braces under axial forces: Parts 1 and 2. In: Summaries of technical papers of annual meeting. Architectural Institute of Japan, pp 1623-1626

Nagao T, Sera S, Nakamura S, Ouchi H, Otani K, Fukutajima K (1992) A study on the RC encased H-section steel brace (Part 1: General description, Part 2: Results and discussion). In: Summaries of Technical Papers of Annual Meeting of the Architectural Institute of Japan, Structural Engineering Section II, Tokyo, Japan: AIJ, pp 1773-1776

Palazzo G, López-Almansa F, Cahís X, Crisafulli F (2009) A low-tech dissipative buckling restrained brace. Design, analysis, production and testing. Eng Struct 31(9):2152-2161. https://doi.org/10.1016/j.engstruct.2009.03.015

Shi Q, Wang F, Wang P, Chen K (2018) Experimental and numerical study of the seismic performance of an allsteel assembled Q195 low-yield buckling-restrained brace. Eng Struct 176:481-499. https://doi.org/10.1016/j.engstruct.2018.09.039

Takeuchi T, Hajjar JF, Matsui R, Nishimoto K, Aiken ID (2010) Local buckling restraint condition for core plates in buckling restrained braces. J Constr Steel Res 66(2):139-149

Timoshenko SP, Gere JM, Prager W (1962) Theory of elastic stability. Journal of Applied Mechanics 29(1):220

Tong J, Guo Y (2017) Global buckling prevention of end collared buckling-restrained braces: Theoretical, numerical analyses and design recommendations. Eng Struct 152:289-306. https://doi.org/10.1016/j.engstruct.2017.09.014

Tong J, Guo Y (2018) Numerical investigations on elastic buckling and hysteretic behavior of steel angles assembled buckling-restrained braces. J Constr Steel Res 144:21-39. https://doi.org/10.1016/j.jcsr.2018.01.015

Tong J, Guo Y, Pan W, Shen M, Zhou P (2020) Global buckling prevention of reduced-core-length bucklingrestrained braces: Theoretical and numerical investigations. B Earthq Eng 18(4):1777-1804. https://doi.org/10.1007/s10518-019-00768-0 
Tremblay R, Bolduc P, Neville R, Devall R (2006) Seismic testing and performance of buckling-restrained bracing systems. Can J Civil Eng 33(2):183-198. https://doi.org/10.1139/L05-103

Yoshino T, Karino Y (1971) Experimental study on shear wall with braces: Part 2. In: Summaries of technical papers of annual meeting. Architectural Institute of Japan, pp 403-404

Zhao J, Lin F, Wang Z (2017) Seismic design of buckling-restrained brace welded end connection considering frame action effects: Theoretical, numerical and practical approaches. Eng Struct 132:761-777. https://doi.org/10.1016/j.engstruct.2016.11.069

Zhao J, Wu B, Ou J (2013) Global stability design method of buckling-restrained braces considering end bending moment transfer: Discussion on pinned connections with collars. Eng Struct 49:947-962. https://doi.org/10.1016/j.engstruct.2012.12.042

\section{5}

\section{Declaration}

536 relationships that could have appeared to influence the work reported in this paper.

\section{Acknowledgments}

538 This study has been supported by research grants from the National Natural Science

539 Foundation of China (52108180) and the Zhejiang Provincial Natural Science Foundation of 540 China (LQ21E080018). 